\title{
Testing the agreement of trees with internal labels
}

\author{
David Fernández-Baca* ${ }^{*}$ and Lei Liu
}

\begin{abstract}
Background: A semi-labeled tree is a tree where all leaves as well as, possibly, some internal nodes are labeled with taxa. Semi-labeled trees encompass ordinary phylogenetic trees and taxonomies. Suppose we are given a collection $\mathcal{P}=\left\{\mathcal{T}_{1}, \mathcal{T}_{2}, \ldots, \mathcal{T}_{k}\right\}$ of semi-labeled trees, called input trees, over partially overlapping sets of taxa. The agreement problem asks whether there exists a tree $\mathcal{T}$, called an agreement tree, whose taxon set is the union of the taxon sets of the input trees such that the restriction of $\mathcal{T}$ to the taxon set of $\mathcal{T}_{i}$ is isomorphic to $\mathcal{T}_{i}$, for each $i \in\{1,2, \ldots, k\}$. The agreement problems is a special case of the supertree problem, the problem of synthesizing a collection of phylogenetic trees with partially overlapping taxon sets into a single supertree that represents the information in the input trees. An obstacle to building large phylogenetic supertrees is the limited amount of taxonomic overlap among the phylogenetic studies from which the input trees are obtained. Incorporating taxonomies into supertree analyses can alleviate this issue.
\end{abstract}

Results: We give $\operatorname{O} \mathcal{O}\left(n k\left(\sum_{i \in[k]} d_{i}+\log ^{2}(n k)\right)\right)$ algorithm for the agreement problem, where $n$ is the total number of distinct taxa in $\mathcal{P}, k$ is the number of trees in $\mathcal{P}$, and $d_{i}$ is the maximum number of children of a node in $\mathcal{T}_{i}$.

Conclusion: Our algorithm can aid in integrating taxonomies into supertree analyses. Our computational experience with the algorithm suggests that its performance in practice is much better than its worst-case bound indicates.

Keywords: Phylogenetic tree, Taxonomy, Agreement, Algorithm

\section{Background}

In the agreement problem, we are given a collection $\mathcal{P}=\left\{\mathcal{T}_{1}, \mathcal{T}_{2}, \ldots, \mathcal{T}_{k}\right\}$ of rooted phylogenetic trees with partially overlapping taxon sets. $\mathcal{P}$ is called a profile and the trees in $\mathcal{P}$ are the input trees. The question is whether there exists a tree $\mathcal{T}$ whose taxon set is the union of the taxon sets of the input trees such that $\mathcal{T}_{i}$ is isomorphic to the restriction of $\mathcal{T}$ to the taxon set of $\mathcal{T}_{i}$, for each $i \in\{1,2, \ldots, k\}$. If such a tree $\mathcal{T}$ exists, then we call $\mathcal{T}$ an agreement tree for $\mathcal{P}$ and say that $\mathcal{P}$ agrees; otherwise, $\mathcal{P}$ disagrees.

Here we study a generalization of the agreement problem, where the internal nodes of the input trees may also be labeled. These labels represent higher-order taxa; that

*Correspondence: fernande@iastate.edu

Department of Computer Science, lowa State University, Ames, IA, USA is, the labels stand for sets of taxa that may nest within each other. Thus, for example, an input tree may contain the taxon Glycine max (soybean) nested within a subtree whose root is labeled Fabaceae (the legumes), containing several other taxa, such as Pisum sativum (pea) and Medicago sativa (alfalfa). The Fabaceae subtree might itself be nested within a subtree whose root is labeled Angiospermae (flowering plants). Note that leaves themselves may be labeled by higher-order taxa. For example, the Fabaceae subtree may contain a leaf labeled Phaseolus, representing the bean genus. Taxonomies are examples of internally labeled trees. A taxonomy groups organisms according to a system of taxonomic rank (e.g., family, genus, and species). Two well-known taxonomies are the NCBI taxonomy [23] and the Angiosperm taxonomy [25]. original author(s) and the source, provide a link to the Creative Commons licence, and indicate if changes were made. The images or other third party material in this article are included in the article's Creative Commons licence, unless indicated otherwise in a credit line to the material. If material is not included in the article's Creative Commons licence and your intended use is not permitted by statutory regulation or exceeds the permitted use, you will need to obtain permission directly from the copyright holder. To view a copy of this licence, visit http://creativecommons.org/licenses/by/4.0/. The Creative Commons Public Domain Dedication waiver (http://creativeco mmons.org/publicdomain/zero/1.0/) applies to the data made available in this article, unless otherwise stated in a credit line to the data. 
We present a $\mathcal{O}\left(n k\left(\sum_{i \in[k]} d_{i}+\log ^{2}(n k)\right)\right)$ algorithm for the agreement problem for trees with internal labels, where $n$ is the total number of distinct taxa in $\mathcal{P}, k$ is the number of trees in $\mathcal{P}$, and, for each $i \in\{1,2, \ldots, k\}, d_{i}$ is the maximum number of children of a node in $\mathcal{T}_{i}$. Our algorithm outputs an agreement supertree for the input trees if such a tree exists; if there is no agreement supertree, the algorithms reports this fact and terminates.

\section{Previous work}

$\mathrm{Ng}$ and Wormald [18] gave the first explicit polynomialtime algorithm for the agreement problem for ordinary rooted phylogenetic trees (i.e., trees without internal labels $)^{1}$. To our knowledge, the fastest algorithm for this problem runs in $O\left(n^{2} k\right)$ time, where $n$ is the number of distinct taxa in $\mathcal{P}$ [11].

The aforementioned algorithms are indebted to Aho et al.s Build algorithm [1], a relative of the agreement problem, the compatibility problem. The input to the compatibility problem is a profile $\mathcal{P}=\left\{\mathcal{T}_{1}, \mathcal{T}_{2}, \ldots, \mathcal{T}_{k}\right\}$ of rooted phylogenetic trees with partially overlapping taxon sets. The question is whether there exists a tree $\mathcal{T}$ whose taxon set is the union of the taxon sets of the input trees such that each input tree $\mathcal{T}_{i}$ can be obtained from the restriction of $\mathcal{T}$ to the taxon set of $\mathcal{T}_{i}$ through edge contractions. If such a tree $\mathcal{T}$ exists, we refer to $\mathcal{T}$ as a compatible tree for $\mathcal{P}$ and say that $\mathcal{P}$ is compatible; otherwise, $\mathcal{P}$ is incompatible.

Compatibility is a less stringent requirement than agreement: any profile that agrees is compatible, but the converse is not true. The compatibility problem for ordinary phylogenetic trees is solvable in $\mathcal{O}\left(M_{\mathcal{P}} \log ^{2} M_{\mathcal{P}}\right)$ time, where $M_{\mathcal{P}}$ is the total number of nodes and edges in the trees of $\mathcal{P}$ [10]. Note that $M_{\mathcal{P}}=\mathcal{O}(n k)$.

Compatibility and agreement reflect two distinct approaches to dealing with multifurcations; i.e., nonbinary nodes, also known as polytomies. Suppose that node $v$ is a multifurcation in some input tree of $\mathcal{P}$ and that $\ell_{1}, \ell_{2}$, and $\ell_{3}$ are taxa in three distinct subtrees of $v$. In an agreement tree for $\mathcal{P}$, these three taxa must be in distinct subtrees of some node in the agreement tree. In contrast, a compatible tree for $\mathcal{P}$ may contain no such node, since a compatible tree is allowed to "refine" the multifurcation at $v$-that is, group two out of $\ell_{1}, \ell_{2}$, and $\ell_{3}$ separately from the third. Thus, compatibility treats multifurcations as "soft" facts; agreement treats them as "hard" facts [17]. Both viewpoints can be valid, depending on the circumstances.

\footnotetext{
${ }^{1}$ These authors refer to what we term "agreement" as "compatibility". What we call "compatibility", they call "weak compatibility".
}

The need for agreement trees to respect the multifurcations in the input trees appears to make testing for agreement harder than testing for compatibility. Indeed, to handle agreement, a costly re-merging step must be added to Build. In this step, certain sets of the taxon partition generated by Build are re-combined to reflect multifurcations $[11,18]$. Similar issues are faced when testing consistency of triples and fans [16]. The situation is more complex for internally labeled trees, because internal nodes with the same label, but in different trees, may jointly imply multifurcations, even if all input trees are binary.

The agreement and compatibility problems are fundamental special cases of the supertree problem, the problem of synthesizing a collection of phylogenetic trees with partially overlapping taxon sets into a single supertree that represents the information in the input trees [2, $5,20,26]$. The original supertree methods were limited to input trees where only the leaves are labeled (that is, ordinary phylogenetic trees), but there has been increasing interest in incorporating internally labeled trees in supertree analysis, motivated by the desire to incorporate taxonomies in these analyses. Taxonomies provide structure and completeness that can be hard to obtain otherwise [14, 19,21], offering a way to circumvent one of the obstacles to building comprehensive phylogenies: the limited taxonomic overlap among different phylogenetic studies [22].

Although internally labeled trees, and taxonomies in particular, are not, strictly speaking, phylogenies, they have many of the same mathematical properties as phylogenies. Both phylogenies and internally labeled trees are X-trees (also called semi-labeled trees) [6, 24]. Nevertheless, algorithmic results for compatibility and agreement of internally labeled trees are scarce, compared to those for ordinary phylogenies. To our knowledge, the first algorithm for testing compatibility of internally labeled trees is in [8] (see also [4]). The fastest known algorithm for the problem runs in $\mathcal{O}\left(M_{\mathcal{P}} \log ^{2} M_{\mathcal{P}}\right)$ time [9]. We are unaware of any previous algorithms for the agreement problem for internally labeled trees.

\section{Organization of the paper}

In the next section ("Preliminaries"), we provide formal definitions of rooted $X$-trees and agreement, as well as a characterization of agreement in terms of lowest common ancestors. We also introduce the display graph, which has a central role in our agreement algorithm. The subsequent section ("Decomposing a Profile") studies the decomposability properties of profiles that agree. These properties allow us to reduce an agreement problem on a profile into independent agreement problems on subprofiles, leading to the agreement algorithm presented 
in the section titled "Constructing an agreement subtree". We report our computational experiences with an implementation of our algorithm in the section titled "Experiments".

\section{Note}

This paper is an extended version of conference paper [13]. The present version contains proofs and has a new section describing our computational experience with an implementation of our algorithm.

\section{Preliminaries}

For each positive integer $r,[r]$ denotes the set $\{1, \ldots, r\}$.

\section{Graphs and trees}

Let $G$ be a graph. $V(G)$ and $E(G)$ denote the node and edge sets of $G$. Let $U$ be a subset of $V(G)$. The subgraph of $G$ induced by $U$ is the graph whose vertex set is $U$ and whose edge set consists of all of the edges in $E(G)$ that have both endpoints in $U$. We write $G \backslash U$ to denote the graph obtained by deleting from $G$ the nodes in $U$, along with their incident edges.

Let $u$ and $v$ be two nodes in $V(G)$. Node $v$ is reachable from $u$ if there exists a path from $u$ to $v$. The connected components of $G$ are the equivalence classes of nodes under the "is reachable from" relation. Let $U$ and $W$ be two subsets of $V(G)$. We say that $U$ and $W$ are disconnected if no node in $W$ is reachable from a node in $U$.

A tree is an acyclic connected graph. All trees here are assumed to be rooted. For a tree $T, r(T)$ denotes the root of $T$. Suppose $u, v \in V(T)$. Then, $u$ is an ancestor of $v$ in $T$, denoted $u \leq_{T} v$, if $u$ lies on the path from $v$ to $r(T)$ in $T$. If $u \leq_{T} v$, then $v$ is a descendant of $u$. Node $u$ is a proper ancestor of $v$, denoted $u<_{T} v$, if $u \leq_{T} v$ and $u \neq v$. We write $u \|_{T} v$ if neither $u \leq_{T} v$ nor $v \leq_{T} u$. If $\{u, v\} \in E(T)$ and $u \leq_{T} v$, then $u$ is the parent of $v$ and $v$ is a child of $u$.

Consider any $x \in V(T)$. We write $\operatorname{parent}_{T}(x)$ and $\mathrm{Ch}_{T}(x)$ to denote the parent of $x$ and the set of children of $x$, respectively. The subtree of $T$ rooted at $x$, denoted $T(x)$, is the subtree of $T$ consisting of all $y \in V(T)$ such that $x \leq_{T} y$. We say that node $x$ is a multifurcation if $\left|\mathrm{Ch}_{T}(x)\right|>2$.

We extend the child notation to subsets of $V(T)$ in the natural way: for $U \subseteq V(T), \mathrm{Ch}_{T}(U)=\bigcup_{u \in U} \mathrm{Ch}_{T}(u)$. Thus, if $U=\emptyset, \mathrm{Ch}_{T}(U)=\emptyset$.

Let $T$ be a tree and suppose $U \subseteq V(T)$. The lowest common ancestor of $U$ in $T$, denoted $\operatorname{LCA}_{T}(U)$, is the unique smallest upper bound of $U$ under $\leq_{T}$. A node $x \in U$ is a minimal node of $T$ in $U$ if for all $y \in U$, either $x \|_{T} y$ or $x \leq_{T} y$. Note that a set $U$ may have multiple minimal nodes and that for any $x \in V(T), x=\operatorname{LCA}_{T}(V(T(x))$ and $x$ is the unique minimal node of $T(x)$ in $V(T(x))$.

\section{Rooted $X$-trees}

Throughout the paper, $X$ denotes a set of labels (that is, taxa, which may be, for instance, species or families of species). A rooted $X$-tree (or $X$-tree, for short), also known as a semi-labeled tree, is a pair $\mathcal{T}=(T, \phi)$ where $T$ is a rooted tree and $\phi$ is a mapping from $X$ to $V(T)$ such that, for every node $v \in V(T)$ with at most one child, $v \in \phi(X) . X$ is the label set of $\mathcal{T}$ and $\phi$ is the labeling function of $\mathcal{T}$. For every node $v \in V(T), \phi^{-1}(v)$ denotes the (possibly empty) subset of $X$ whose elements map into $v$; these elements as the labels of $v$. If $\phi^{-1}(v) \neq \emptyset$, then $v$ is labeled; otherwise, $v$ is unlabeled. For $U \subseteq V(T)$, we write $\phi^{-1}(U)$ to denote $\bigcup_{u \in U} \phi^{-1}(u)$.

By definition, every leaf in an $X$-tree is labeled, and any node, including the root, that has a single child must be labeled. Nodes with two or more children may be labeled or unlabeled. An $X$-tree $\mathcal{T}=(T, \phi)$ is singularly labeled if every node in $T$ has at most one label; $\mathcal{T}$ is fully labeled if every node in $T$ is labeled.

$X$-trees generalize ordinary phylogenetic trees (also known as phylogenetic X-trees [24]). An ordinary phylogenetic tree is a semi-labeled tree $\mathcal{T}=(T, \phi)$ where $r(T)$ has at least two children and $\phi$ is a bijection from $X$ into leaf set of $T$ (thus, internal nodes are not labeled).

Let $\mathcal{T}=(T, \phi)$ be an $X$-tree. For each $u \in V(T)$, $X(u)$ denotes the set of all labels in $T(u)$; that is, $X(u)=\bigcup_{v: u \leq T} \phi^{-1}(v) . X(u)$ is called a cluster of $T$. $\mathrm{Cl}(\mathcal{T})$ denotes the set of all clusters of $\mathcal{T}$. We extend the cluster notation to sets of nodes as follows. Let $U$ be a subset of $V(T)$. Then, $X(U)=\bigcup_{v \in U} X(v)$. If $U=\emptyset$, then $X(U)=\emptyset$.

Suppose $\quad Y \subseteq X \quad$ for an $X$-tree $\mathcal{T}=(T, \phi)$. The restriction of $\mathcal{T}$ to $Y$, denoted $\mathcal{T} \mid Y$, is the semi-labeled tree whose cluster set is $\mathrm{Cl}(\mathcal{T} \mid Y)=\{W \cap Y: W \in \mathrm{Cl}(\mathcal{T})$ and $W \cap Y \neq \emptyset\}$.

Intuitively, $\mathcal{T} \mid Y$ is obtained from the minimal rooted subtree of $T$ that connects the nodes in $\phi(Y)$ by suppressing all vertices $v$ such that $v \notin \phi(Y)$ and $v$ has only one child.

Let $\mathcal{T}=(T, \phi)$ be an $X$-tree and $\mathcal{T}^{\prime}=\left(T^{\prime}, \phi^{\prime}\right)$ be an $X^{\prime}$-tree such that $X^{\prime} \subseteq X . \mathcal{T}$ agrees with $\mathcal{T}^{\prime}$ if $\mathrm{Cl}\left(\mathcal{T}^{\prime}\right)=\operatorname{Cl}\left(\mathcal{T} \mid X^{\prime}\right)$. It is well known that the clusters of a tree determine the tree, up to isomorphism [24, Theorem 3.5.2]. Thus, $\mathcal{T}$ agrees with $\mathcal{T}^{\prime}$ if $\mathcal{T}^{\prime}$ and $\mathcal{T} \mid X^{\prime}$ are isomorphic.

\section{Profiles and agreement}

Throughout the rest of this paper, $\mathcal{P}$ denotes a set $\left\{\mathcal{T}_{1}, \mathcal{T}_{2}, \ldots, \mathcal{T}_{k}\right\}$ such that, for each $i \in[k], \mathcal{T}_{i}=\left(T_{i}, \phi_{i}\right)$ is an $X_{i}$-tree for some label set $X_{i}$ (Fig. 1). We refer to $\mathcal{P}$ as a profile, and to the trees in $\mathcal{P}$ as input trees. We write $X_{\mathcal{P}}$ to denote $\bigcup_{i \in[k]} X_{i}$. 

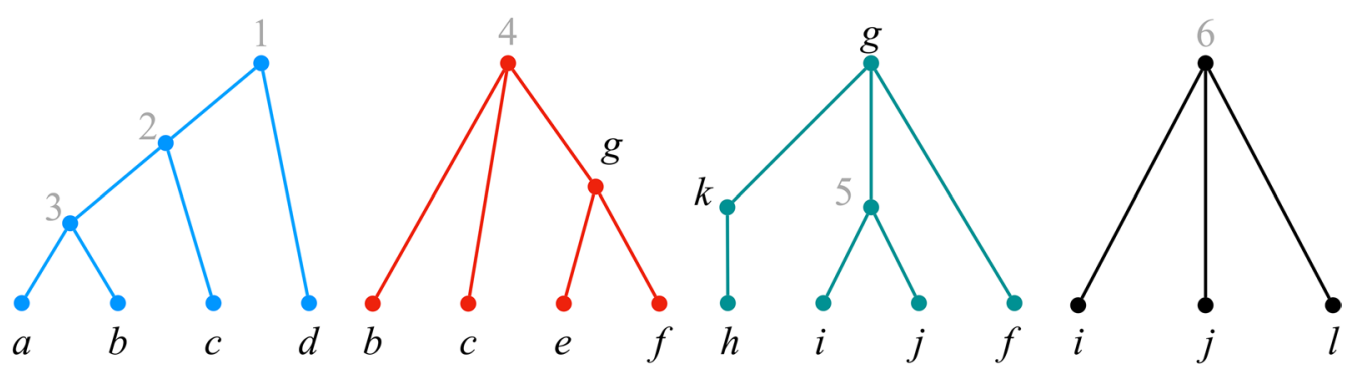

Fig. 1 A profile $\mathcal{P}=\left\{\mathcal{T}_{1}, \mathcal{T}_{2}, \mathcal{T}_{3}, \mathcal{T}_{4}\right\}$. The letters are the original labels; grey numbers are labels added to make the trees fully labeled. We use this profile as a running example throughout the paper

A profile $\mathcal{P}$ agrees if there is an $X_{\mathcal{P}}$-tree $\mathcal{T}$ that agrees with each of the trees in $\mathcal{P}$. If $\mathcal{T}$ exists, we refer to $\mathcal{T}$ as an agreement tree for $\mathcal{P}$. See Fig. 2.

Given a subset $Y$ of $X_{\mathcal{P}}$, the restriction of $\mathcal{P}$ to $Y$, denoted $\mathcal{P} \mid Y$, is the profile defined as $\mathcal{P} \mid Y=\left\{\mathcal{T}_{1}\left|Y \cap X_{1}, \mathcal{T}_{2}\right| Y \cap X_{2}, \ldots, \mathcal{T}_{k} \mid Y \cap X_{k}\right\}$

Lemma 1 Suppose a profile $\mathcal{P}$ has an agreement tree $\mathcal{T}$ . Then, for any $Y \subseteq X_{\mathcal{P}}, \mathcal{T} \mid Y$ is an agreement tree for $\mathcal{P} \mid Y$.

Proof Consider any $i \in[k]$. Since $\mathcal{T}$ agrees with $\mathcal{T}_{i}$, $A \in \operatorname{Cl}\left(\mathcal{T}_{i}\right)$ if and only if $A \in \operatorname{Cl}\left(\mathcal{T} \mid X_{i}\right)$. Thus, for any $Y \subseteq X_{\mathcal{P}}$, if $Y_{i}=Y \cap X_{i}$, then $A \cap Y_{i} \in \operatorname{Cl}\left(\mathcal{T}_{i} \mid Y_{i}\right)$ if and only if $A \cap Y \in \operatorname{Cl}\left(\mathcal{T} \mid Y_{i}\right)$. The lemma follows.

We can convert a profile $\mathcal{P}$ containing trees that are not fully labeled into an equivalent profile $\mathcal{P}^{\prime}$ of fully-labeled trees as follows. For each $i \in[k]$, let $l_{i}$ be the number of unlabeled nodes in $T_{i}$. Create a set $X^{\prime}$ of $n^{\prime}=\sum_{i \in[k]} l_{i}$ labels such that $X^{\prime} \cap X_{\mathcal{P}}=\emptyset$. For each $i \in[k]$ and each $v \in V\left(T_{i}\right)$ such that $\phi_{i}^{-1}(v)=\emptyset$, make $\phi_{i}^{-1}(v)=\{\ell\}$, where $\ell$ is a distinct element from $X^{\prime}$. We refer to $\mathcal{P}^{\prime}$ as the profile obtained by adding distinct new labels to $\mathcal{P}$. See Fig. 1.

The proof of the next result is analogous to that of $[8$, Lemma 3.4].

Lemma 2 Let $\mathcal{P}^{\prime}$ be the profile obtained by adding distinct new labels to $\mathcal{P}$. Then, $\mathcal{P}$ agrees if and only if $\mathcal{P}^{\prime}$ agrees. Further, if $\mathcal{T}$ is an agreement tree for $\mathcal{P}^{\prime}$, then $\mathcal{T}$ is also an agreement tree for $\mathcal{P}$.

Proof Let $\mathcal{P}^{\prime}=\left\{\mathcal{T}_{1}^{\prime}, \mathcal{T}_{2}^{\prime}, \ldots, \mathcal{T}_{k}^{\prime}\right\}$. For each $i \in[k]$, let $X_{i}^{\prime}$ be the set of new labels added to $\mathcal{T}_{i}$ to obtain $\mathcal{T}_{i}^{\prime}$. By definition, if $\mathcal{T}$ is an agreement tree for $\mathcal{P}^{\prime}$, then, for each $i \in[k], A \in \mathrm{Cl}\left(\mathcal{T}_{i}^{\prime}\right)$ if and only if $A \in \mathrm{Cl}\left(\mathcal{T} \mid\left(X_{i} \cup X_{i}^{\prime}\right)\right)$. To prove the lemma, it suffices now to show that, for each $i \in[k], A \in \mathrm{Cl}\left(\mathcal{T}_{i}^{\prime}\right)$ if and only if $A \cap X_{i} \in \mathrm{Cl}\left(\mathcal{T}_{i}\right)$. We omit the details. $\square$
From this point forward, we make the following assumption.

Assumption 1 For each $i \in[k], \mathcal{T}_{i}$ is fully and singularly labeled.

Lemma 2 implies that no generality is lost in assuming that all trees in $\mathcal{P}$ are fully labeled. Note that even if the trees in $\mathcal{P}$ are singularly labeled, a tree that agrees with $\mathcal{P}$ is not necessarily singularly labeled. See Fig. 2.

By Assumption 1, for each $i \in[k]$, there is a bijection between the labels in $X_{i}$ and the nodes of $V\left(T_{i}\right)$. (As noted earlier, however, if $\mathcal{T}=(T, \phi)$ is an agreement tree for $\mathcal{P}$, then $\phi$ is not in general a bijection between $X_{\mathcal{P}}$ and $V(T)$.) For this reason, we will often refer to nodes of the input trees by their labels. In particular, given a label $\ell \in X_{i}$, we write $X_{i}(\ell)$ to denote $X_{i}\left(\phi_{i}(\ell)\right)$ (the cluster of $\mathcal{T}_{i}$ at the node labeled $\ell$ ), $\mathrm{Ch}_{T_{i}}(\ell)$ to denote $\phi_{i}\left(\mathrm{Ch}_{T_{i}}\left(\phi_{i}(\ell)\right)\right.$ (the labels of children of $\ell$ in $\left.\mathcal{T}_{i}\right)$, and $\mathrm{Ch}_{T_{i}}(A)$ to denote $\phi_{i}^{-1}\left(\mathrm{Ch}_{T_{i}}\left(\phi_{i}(A)\right)\right.$, for $A \subseteq X_{i}$.

The following characterization of agreement generalizes a result in [11].

Lemma 3 Let $\mathcal{P}$ be a profile and $\mathcal{T}=(T, \phi)$ be an $X_{\mathcal{P}}$ -tree. Then, $\mathcal{T}$ is an agreement tree for $\mathcal{P}$ if and only if for each $i \in[k]$ and each label $a \in X_{i}$,

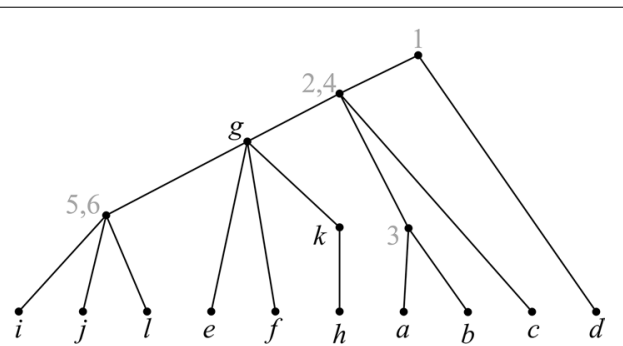

Fig. 2 An agreement tree for $\mathcal{P}$. Although all input trees are singularly labeled, the agreement tree is not 
(E1) $\phi(a)=\operatorname{LCA}_{T}\left(X_{i}(a)\right)$,

(E2) for each label $b \in \mathrm{Ch}_{T_{i}}(a), \phi(a)<_{T} \phi(b)$, and

(E3) for every two distinct labels $b, c \in \mathrm{Ch}_{T_{i}}$ (a), there exist distinct nodes $u, v \in \mathrm{Ch}_{T}(\phi(a))$ such that $\phi(b) \in X_{\mathcal{P}}(u)$ and $\phi(c) \in X_{\mathcal{P}}(v)$.

Proof (If) Suppose that $\phi$ satisfies conditions (E1)(E3). To prove that $\mathcal{T}$ agrees with $\mathcal{T}_{i}$, we show that $\mathrm{Cl}\left(\mathcal{T}_{i}\right)=\mathrm{Cl}\left(\mathcal{T} \mid X_{i}\right)$

First, we show that $\mathrm{Cl}\left(\mathcal{T}_{i}\right) \subseteq \mathrm{Cl}\left(\mathcal{T} \mid X_{i}\right)$ by arguing that $X_{i}(a)=X_{\mathcal{P}}(\phi(a)) \cap X_{i}$, for each $a \in X_{i}$. By (E1), $\quad X_{i}(a) \subseteq X_{\mathcal{P}}(\phi(a))$. Now, suppose that there is a label $b \in X_{\mathcal{P}}(\phi(a)) \cap X_{i}$ such that $b \notin X_{i}(a)$. Let $c=\operatorname{LCA}_{T_{i}}\left(X_{i}(a) \cup\{b\}\right)$. Then, since $b \notin X_{i}(a)$, $X_{i}(a) \subset X_{i}(c)$. Hence, $c<T_{i} a$ and, by (E2), $\phi(c)<_{T} \phi(a)$. Thus, (i) there exist distinct labels $d, d^{\prime} \in \mathrm{Ch}_{T_{1}}(c)$ such that $a \in X_{i}(d)$ and $b \in X_{i}\left(d^{\prime}\right)$. but (ii) since $a, b \in X_{\mathcal{P}}(\phi(a)) \cap X_{i}$, there is a single child $u$ of $\mathrm{Ch}_{\mathcal{P}}(c)$ such that $a, b \in X_{\mathcal{P}}(v)$, contradicting condition (E3).

Next, we prove that $\mathrm{Cl}\left(\mathcal{T} \mid X_{i}\right) \subseteq \mathrm{Cl}\left(\mathcal{T}_{i}\right)$. Suppose, to the contrary, that there is a cluster $Y \in \mathrm{Cl}\left(\mathcal{T} \mid X_{i}\right) \backslash \mathrm{Cl}\left(\mathcal{T}_{i}\right)$. Let $u=\operatorname{LCA}_{T}(Y)$; thus, $Y=X_{\mathcal{P}}(u) \cap X_{i}$. Let $a=\operatorname{LCA}_{T_{i}}(Y)$. Then, $Y \subset X_{i}(a)$. Choose any $b \in X_{i}(a) \backslash Y$; thus, $a \leq_{T_{i}} b$. Note that $b \notin X_{\mathcal{P}}(u)$ and $\phi(a)<_{T} u$. We have two cases.

(i) $a \neq b$. Then, $a<_{i} b$. On the other hand, we have either $\phi(a) \geq_{T} \phi(b)$ or $\phi(a) \|_{T} \phi(b)$, contradicting (E2).

(ii) $a=b$. Then, there exist distinct labels $c_{1}, c_{2} \in \mathrm{Ch}_{T_{i}}(a)$ such that $Y \cap X_{i}\left(c_{j}\right) \neq \varnothing$ and $Y \nsubseteq X_{i}\left(c_{j}\right)$, for $j \in[2]$. By (E1), $\phi(a)=\operatorname{LCA}_{T}\left(X_{i}(a)\right)$. Since $\phi(a)<_{T} u$, there exists a unique node $v \in \mathrm{Ch}_{T}(u)$ such that $Y \subset X_{\mathcal{P}}(v)$. But then $\phi\left(c_{1}\right)$ and $\phi\left(c_{2}\right)$ descend from the same child, $v$, of $\phi(a)$, contradicting condition (E3).

(Only if) Suppose that $\mathcal{T}$ agrees with $\mathcal{T}_{i}$. It is straightforward to show that $\phi$ must satisfy (E1). Thus, we focus on conditions (E2) and (E3).

Suppose condition (E2) does not hold. Then, there exists a label $b \in \mathrm{Ch}_{T_{i}}(a)$, such that $\phi(a) \geq_{T} \phi(b)$. Since $X_{\mathcal{P}}(\phi(b)) \neq X_{\mathcal{P}}(\phi(a))$, we must have $\phi(a)>_{T} \phi(b)$. But then $\mathcal{T}$ does not agree with $\mathcal{T}_{i}$, a contradiction.

Suppose condition (E3) does not hold. Then, there exist distinct labels $c, c^{\prime} \in \mathrm{Ch}_{T_{i}}(a)$ such that $\left\{\phi(c), \phi\left(c^{\prime}\right)\right\} \subseteq X_{\mathcal{P}}(v)$, for some $v \in \mathrm{Ch}_{T}(\phi(a))$. But then $\mathcal{T} \mid X_{i}$ contains cluster $Y=X_{\mathcal{P}}(v) \cap X_{i}$, which is not in $\mathcal{T}_{i}$, contradicting the assumption that $\mathcal{T}$ agrees with $\mathcal{T}_{i}$.
Lemma 4 If profile $\mathcal{P}$ agrees, then $\mathcal{P}$ has an agreement tree $\mathcal{T}=(T, \phi)$ such that $\phi^{-1}(v) \neq \emptyset$ for each node $v \in V(T)$.

Proof Suppose there is a node $v \in V(T)$ such that $\phi^{-1}(v)=\emptyset$. Note that $v$ cannot be a leaf. Let $u_{1}, u_{2}, \ldots, u_{d}$ be the children of $v$. We use the following fact.

Fact. For each $i \in[k]$, there is at most one $j \in[d]$ such that $X_{\mathcal{P}}\left(u_{j}\right) \cap X_{i} \neq \emptyset$.Proof Assume to the contrary that there exist distinct $j, j^{\prime} \in[d]$ such that $W=X_{\mathcal{P}}\left(u_{j}\right) \cap X_{i} \neq \emptyset \quad$ and $\quad W^{\prime}=X_{\mathcal{P}}\left(u_{j^{\prime}}\right) \cap X_{i} \neq \emptyset$. Let $c=\mathrm{LCA}_{T_{i}}(W)$ and $c^{\prime}=\mathrm{LCA}_{T_{i}}\left(W^{\prime}\right)$ and let $a=\mathrm{LCA}_{T_{i}}\left(W \cup W^{\prime}\right)$. Then, $c$ and $c^{\prime}$ are in distinct subtrees of $T_{i}(a)$. By Lemma 3, $\phi(c)$ and $\phi\left(c^{\prime}\right)$ are in distinct subtrees of $v$ and $v=\phi(a)$. But this contradicts the assumption that $\phi^{-1}(v)=\emptyset$.

Now, choose any $j \in[d]$. Let $T^{\prime}$ be the tree obtained by contracting the edge $\left(v, u_{j}\right) \in E(T)$. That is, $T^{\prime}$ is obtained by eliminating edge $\left(v, u_{j}\right)$, deleting $u_{j}$, and making $\mathrm{Ch}_{T^{\prime}}(v)=\mathrm{Ch}_{T}(v) \cup \mathrm{Ch}_{T}\left(u_{j}\right)$. Let $\mathcal{T}^{\prime}=\left(T^{\prime}, \phi^{\prime}\right)$, where $\left(\phi^{\prime}\right)^{-1}(w)=\phi^{-1}(w)$, if $w \in V(T) \backslash\left\{v, u_{j}\right\}$, and $\left(\phi^{\prime}\right)^{-1}(v)=\phi^{-1}(v) \cup \phi^{-1}\left(u_{j}\right)$. Then, the above fact implies that, for each $i \in[k], \mathrm{Cl}\left(\mathcal{T} \mid X_{i}\right)=\mathrm{Cl}\left(\mathcal{T}^{\prime} \mid X_{i}\right)$. That is, $\mathcal{T}^{\prime}$ is also an agreement tree for $\mathcal{P}$. Let $\mathcal{T}^{\prime \prime}=\left(T^{\prime \prime}, \phi^{\prime \prime}\right)$ be the tree that results from repeating this contraction operation until it no longer applies. Then, $\mathcal{T}^{\prime \prime}$ satisfies $\left(\phi^{\prime \prime}\right)^{-1}(v) \neq \emptyset$ for each node $v \in V\left(T^{\prime \prime}\right)$.

\section{The display graph}

The display graph of a profile $\mathcal{P}$, denoted $H_{\mathcal{P}}$, is the graph obtained from the disjoint union of the underlying trees $T_{1}, \ldots, T_{k}$ of $\mathcal{P}$ by identifying nodes that have the same label (parallel edges are replaced by a single edge) $[7,9$, 10]. See Fig. 3 . As we shall see, $H_{\mathcal{P}}$ plays a major role in our agreement algorithm.

$H_{\mathcal{P}}$ has $O(n k)$ nodes and edges, and can be constructed in $O(n k)$ time. By Assumption 1, there is a bijection between the labels in $X$ and the nodes of $H_{\mathcal{P}}$. Thus, from this point forward, we refer to the nodes of $H_{\mathcal{P}}$ by their labels.

\section{Decomposing a profile}

A position in a profile $\mathcal{P}$ is a tuple $\pi=\left(\pi_{1}, \pi_{2}, \ldots, \pi_{k}\right)$ where $\pi_{i} \subseteq X_{i}$, for each $i \in[k]$. At any given point during its execution, our agreement algorithm focuses on testing the agreement of the subprofile of $\mathcal{P}$ determined by the subtrees associated with a specific position. 


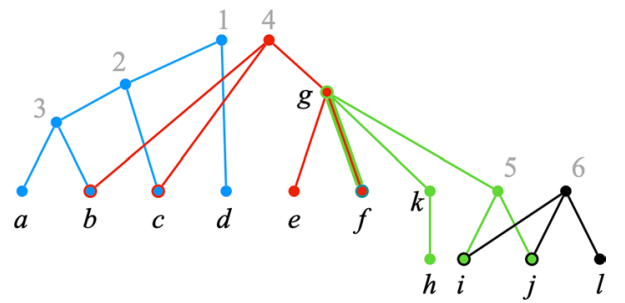

Fig. 3 Display graph. The display graph $H_{\mathcal{P}}$ of the profile of Fig. 1

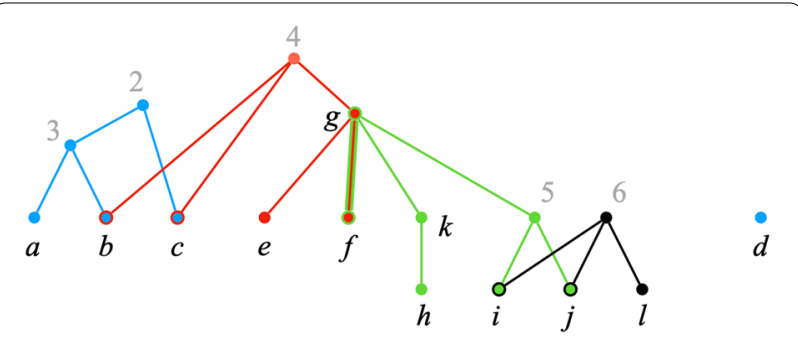

Fig. 4 A decomposition of $\pi^{\text {init }} \cdot H_{\mathcal{P}} \backslash\{1\}$. Each connected component corresponds to a distinct successor position of $\pi^{\text {init }}$

Proof (Only if) Suppose $\mathcal{P}$ has an agreement tree $\mathcal{T}$. For any valid position $\pi$ in $\mathcal{P}, X_{\mathcal{P}}(\pi) \subseteq X_{\mathcal{P}}$. Thus, by Lemma $1, \mathcal{T} \mid X_{\mathcal{P}}(\pi)$ is an agreement tree for $\pi$.

(If) Suppose there is an agreement tree for every valid position $\pi$ in $\mathcal{P}$. Then, in particular, there exists an agreement tree $\mathcal{T}$ for the initial position $\pi^{\text {init }}$ of $\mathcal{P}$. Since $X_{\mathcal{P}}\left(\pi^{\text {init }}\right)=X_{\mathcal{P}}, \mathcal{T}$ must also be an agreement tree for $\mathcal{P}$. even if $\ell \in X_{i}$ and $\ell \in X_{j}$. Thus, for example, in the profile of Fig. 1 , we have $g \in \pi_{3}^{\text {init }}$, but $g \notin \pi_{2}^{\text {init }}$, even though $g$ appears in trees $T_{3}$ and $T_{2}$.

For a position $\pi$ in $\mathcal{P}$, let $X_{\mathcal{P}}(\pi)$ denote the set of labels $\bigcup_{i \in[k]} X_{i}\left(\pi_{i}\right) . H_{\mathcal{P}}(\pi)$ denotes the subgraph of $H_{\mathcal{P}}$ induced by $X_{\mathcal{P}}(\pi)$. Thus, $H_{\mathcal{P}}\left(\pi^{\text {init }}\right)=H_{\mathcal{P}}$.

A position $\pi$ in $\mathcal{P}$ is valid if, for each $i \in[k]$,

$$
\pi_{i}= \begin{cases}\left\{\operatorname{LCA}_{T_{i}}\left(X_{i} \cap X_{\mathcal{P}}(\pi)\right)\right\} & \text { if } X_{i} \cap X_{\mathcal{P}}(\pi) \neq \emptyset, \\ \emptyset, & \text { otherwise }\end{cases}
$$

Thus, if $\pi$ is valid, then, for each $i \in[k]$ such that $X_{i} \cap X_{\mathcal{P}}(\pi) \neq \emptyset$, component $\pi_{i}$ consists of a single label $\ell$ such that $T_{i}(\ell)$ contains every label in $H_{\mathcal{P}}(\pi)$ that also belongs to $X_{i}$. Clearly, $\pi^{\text {init }}$ is a valid position.

Let $\pi$ be a valid position. A label $\ell \in \bigcup_{i \in[k]} \pi_{i}$ is exposed in $\pi$ if $\pi_{i}=\{\ell\}$ for every $i \in[k]$ such that $\ell \in X_{i} \cap X_{\mathcal{P}}(\pi)$. A set $S \subseteq \bigcup_{i \in[k]} \pi_{i}$ is an exposed subset in $\pi$ (exposed subset for short, when $\pi$ is understood) if every label $\ell \in S$ is exposed.

Consider the initial position $\pi^{\text {init }}$ of the profile of Fig. 1. Label 1 is exposed in $\pi^{\text {init }}$ since $\pi_{1}^{\text {init }}=\{1\}$ and label 1 exists only in $\mathcal{T}_{1}$. Similarly, labels 4 and 6 are both exposed. On the other hand, label $g$ is not exposed, since it appears in trees $T_{2}$ and $T_{3}$, but $g \notin \pi_{2}^{\text {init }}$, even though $\pi_{3}^{\text {init }}=\{g\}$.

We say that a position $\pi$ has an agreement tree if $\mathcal{P} \mid X_{\mathcal{P}}(\pi)$ has an agreement tree.

Lemma 5 A profile $\mathcal{P}$ has an agreement tree if and only if there exists an agreement tree for every valid position $\pi$ in $\mathcal{P}$.

\section{Decomposing a position}

In what follows, $\pi$ denotes a valid position in $\mathcal{P}$. For each $i \in[k]$ such that $\pi_{i} \neq \emptyset, \ell_{i} \in X_{i}$ denotes the single label in $\pi_{i}$.

Let $S$ be an exposed subset of $\pi$. We say that $S$ is nice if for each connected component $W$ of $H_{\mathcal{P}}(\pi) \backslash S$, the position $\pi^{W}=\left(\pi_{1}^{W}, \pi_{1}^{W}, \ldots, \pi_{k}^{W}\right)$ defined as follows is valid:

$$
\pi_{i}^{W}=\left\{a: a \text { is a minimal label of } T_{i} \text { in } X_{i} \cap W\right\} .
$$

Observe that if $S$ is nice, $W=X_{\mathcal{P}}\left(\pi^{W}\right)$, for each connected component $W$ of $H_{\mathcal{P}}(\pi) \backslash S$.

If $S$ is a nice exposed set, we refer to the set $\left\{\pi^{W}: W\right.$ is a connected component of $\left.H_{\mathcal{P}}(\pi) \backslash S\right\}$ of valid positions as the successor positions of $\pi$ (with respect to $S$ ).

A good decomposition of $\pi$ is a pair $(S, \Pi)$, where $S$ is a nice exposed subset and $\Pi$ is the collection of successor positions of $\pi$ with respect to $\pi$. Note that $X_{\mathcal{P}}(\pi)=S \cup \bigcup_{\pi^{\prime} \in \Pi} X_{\mathcal{P}}\left(\pi^{\prime}\right)$. Note also that we allow $S$ or $\Pi$ to be empty.

Consider the profile of Fig. 1, whose display graph is in Fig. 3. Let $S=\{1\}$. Figure 4 shows $H_{\mathcal{P}}\left(\pi^{\text {init }}\right) \backslash S$. $H_{\mathcal{P}}(\pi) \backslash S$ has two connected components, $W_{1}=\{d\}$ and $W_{2}=\{2,3,4,5,6, a, b, c, e, f, g, h, i, j, k, l\}$. Then, by Eq. (2), the corresponding positions are

$$
\pi^{W_{1}}=(\{d\}, \emptyset, \emptyset, \emptyset) \text { and } \pi^{W_{2}}=(\{2\},\{4\},\{g\},\{6\}) .
$$


Positions $\pi^{W_{1}}$ and $\pi^{W_{2}}$ are clearly valid positions. Therefore, $S$ is nice and $(S, \Pi)$, where $\Pi=\left\{\pi^{W_{1}}, \pi^{W_{2}}\right\}$, is a good decomposition of $\pi^{\text {init }}$.

The next result is central to our agreement algorithm.

Lemma 6 Let $\pi$ be a valid position in a profile $\mathcal{P}$. Then, $\pi$ has an agreement tree if and only if there exists a good decomposition $(S, \Pi)$ of $\pi$ such that $S \neq \emptyset$ and, for each position $\pi^{\prime} \in \Pi, \pi^{\prime}$ has an agreement tree. If such a good decomposition exists, then $\pi$ has an agreement tree $\mathcal{T}=(T, \phi)$ where $\phi^{-1}(r(T))=S$.

Proof (Only if) Suppose position $\pi$ has an agreement tree $\mathcal{T}=(T, \phi)$ (thus, $\mathcal{T}$ is an $X_{\mathcal{P}}(\pi)$-tree). Let $S=\phi^{-1}(r(T))$. By Lemma 4 , we can assume that $S \neq \emptyset$. Note that every label $\ell \in S$ must be in $\bigcup_{i \in[k]} \pi_{i}$. Further, $\ell$ must be exposed in $\pi$. Indeed, if $\ell$ is not exposed, there exists an $i \in[k]$ such that $\pi_{i} \neq\left\{\ell^{\prime}\right\}$, where $\ell^{\prime}<_{T_{i}} \ell$, so $\ell \notin \phi^{-1}(r(T))$, a contradiction.

If $T$ consists of a single node $u=r(T)$, then we must have $S=\bigcup_{i \in[k]} \pi_{i}=X_{\mathcal{P}}(\pi)$. Then, $(S, \emptyset)$ is trivially a good decomposition of $\pi$.

Now, suppose $\mathrm{Ch}_{T}(r(T))=\left\{v_{1}, v_{2}, \ldots, v_{d}\right\}$, where $d \geq 1$. For each $j \in[d]$, let $\mathcal{T}^{(j)}=\mathcal{T} \mid X_{\mathcal{P}}\left(v_{j}\right)$. By Lemma $1, \mathcal{T}^{(j)}$ is an agreement tree for $\mathcal{P} \mid X_{\mathcal{P}}\left(v_{j}\right)$. For each $i \in[k]$ and each $j \in[d]$ such that $X_{i} \cap X_{\mathcal{P}}\left(v_{j}\right) \neq \emptyset$, let

$$
\ell_{i}^{(j)}=\operatorname{LCA}_{T_{i}}\left(X_{i} \cap X_{\mathcal{P}}\left(v_{j}\right)\right) .
$$

Thus, $\ell_{i}^{(j)}$ is the root of $\mathcal{T}_{i} \mid\left(X_{i} \cap X_{\mathcal{P}}\left(v_{j}\right)\right)$.

For each $j \in[d]$, define a position $\pi^{(j)}$, where, for each $i \in[k]$,

$$
\pi_{i}^{(j)}= \begin{cases}\left\{\ell_{i}^{(j)}\right\} & \text { if } X_{i} \cap X_{\mathcal{P}}\left(v_{j}\right) \neq \varnothing \\ \varnothing & \text { otherwise. }\end{cases}
$$

Let $\Pi=\left\{\pi^{(1)}, \pi^{(2)}, \ldots, \pi^{(d)}\right\}$. By construction, for each $j \in[d]$, $\pi^{(j)}$ satisfies Eq. (1), so $\pi^{(j)}$ is valid. Since $X_{\mathcal{P}}\left(v_{j}\right)=X_{\mathcal{P}}\left(\pi^{(j)}\right), \mathcal{T}^{(j)}$ is an agreement tree for $\pi^{(j)}$.

For any $j^{\prime} \in[d]$ such that $j^{\prime} \neq j, X_{\mathcal{P}}\left(\pi^{(j)}\right)$ and $X_{\mathcal{P}}\left(\pi^{\left(j^{\prime}\right)}\right)$ are disconnected in $H_{\mathcal{P}}(\pi) \backslash S$, since every path between the two sets must go through a label in $S$. Note, however, that $X_{\mathcal{P}}\left(\pi^{(j)}\right)$ may contain multiple connected components of $H_{\mathcal{P}}(\pi) \backslash S$. For each connected component $W$ of $H_{\mathcal{P}}\left(\pi^{(j)}\right)$, let $\mathcal{T}^{(j, W)}=\mathcal{T}^{(j)} \mid W$ and let $\pi^{(j, W)}$ be the position where $\pi_{i}^{(j, W)}=\pi_{i}^{(j)} \cap W$, for each $i \in[k]$. Then, $\mathcal{T}^{(j, W)}$ is an agreement tree for $\pi^{(j, W)}$.
Let $\Pi$ consist of all positions $\pi^{(j, W)}$ such that $j \in[d]$ and $W$ is a connected component of $H_{\mathcal{P}}\left(\pi^{(j)}\right)$. Then $(S, \Pi)$ is a good decomposition of $\pi$, where each position in $\Pi$ has an agreement tree.

(If) Let $(S, \Pi)$ be a good decomposition of $\pi$ such that $S \neq \emptyset$ and each position in $\Pi$ has an agreement tree. If $\Pi=\emptyset$, then we must have $S=X_{\mathcal{P}}(\pi)$. Further, for each $i \in[k]$ such that $\pi_{i} \neq \emptyset$, it must be the case that $T_{i}$ consists of a single node, labeled by the single label in $\pi_{i}$. Let $T$ be the tree consisting of a single node $u=r(T)$ and let $\phi(\ell)=u$, for all $u \in S$. Then, $\mathcal{T}=(T, \phi)$ is an agreement tree for $\pi$.

Now suppose $\Pi \neq \emptyset$. Let $\Pi=\left\{\pi^{(1)}, \pi^{(2)}, \ldots, \pi^{(d)}\right\}$. For each $j \in[d]$, let $\mathcal{T}^{(j)}=\left(T^{(j)}, \phi^{(j)}\right)$ be an agreement tree for $\pi^{(j)}$, and let $v_{j}$ be the root of $T^{(j)}$. Let $\mathcal{T}=(T, \phi)$ be the $X_{\mathcal{P}}(\pi)$-tree where $T$ is assembled by creating a new node $u$ and making $\mathrm{Ch}_{T}(u)=\left\{v_{1}, v_{2}, \ldots, v_{d}\right\}$ and, for each $\ell \in X_{\mathcal{P}}(\pi), \phi(\ell)$ is defined as

$$
\phi(\ell)= \begin{cases}u & \text { if } \ell \in S \\ \phi^{(j)}(\ell) & \text { if } \ell \in X_{\mathcal{P}}\left(\pi^{(j)}\right) .\end{cases}
$$

Since $(S, \Pi)$ is a good decomposition, $S \cup \bigcup_{j \in[k]} X_{\mathcal{P}}\left(\pi^{(j)}\right)=X_{\mathcal{P}}(\pi)$. Thus, $\mathcal{T}$ is an $X_{\mathcal{P}}(\pi)$-tree. We prove that $\mathcal{T}$ is an agreement tree for $\pi$, by showing that, for each $i \in[k], \phi$ satisfies properties (E1)-(E3) of Lemma 3.

By Lemma 3 every label in $\mathcal{T}_{i} \mid X_{i}\left(\pi_{i}^{(j)}\right)$ satisfies (E1)(E3). For each $j \in[d]$, let $\ell_{i}$ be the label of the root of $\mathcal{T}_{i} \mid X_{i}\left(\pi_{i}^{(j)}\right)$. There are two possibilities:

(i) $\ell_{i} \in \phi^{-1}(u)$. Then, each of $\ell_{i}$ s children must be in a distinct subtree of $u$. Thus, properties (E1)-(E3) are satisfied.

(ii) $\ell_{i} \notin \phi^{-1}(u)$. Then, $\ell_{i}$ and all of its children must be contained in a single subtree, say $\mathcal{T}_{j}$, of $u$, and the claim follows from the fact that $\phi^{(j)}$ satisfies properties (E1)-(E3).

\section{Good partitions}

To find a good decomposition of a position $\pi$, it is convenient to work with partitions of the set of children of the labels in $\pi$. We write $\mathrm{Ch}_{\mathcal{P}}(\pi)$ to denote the set of all children of some label in $\pi$; i.e., $\mathrm{Ch}_{\mathcal{P}}(\pi)=\bigcup_{i \in[k]} \mathrm{Ch}_{T_{i}}\left(\pi_{i}\right)$ 
Let $S$ be an exposed subset of $\pi$. The partition of $\mathrm{Ch}_{\mathcal{P}}(\pi)$ induced by $S$, denoted $\Psi(S)$, is the set consisting of all $A \subseteq \mathrm{Ch}_{\mathcal{P}}(\pi)$ such that $A=\mathrm{Ch}_{\mathcal{P}}(\pi) \cap W$ for some connected component $W$ of $H_{\mathcal{P}}(\pi) \backslash S$

Lemma 7 Let $S$ be a subset of the exposed nodes in a valid position $\pi$. S is a nice set for $\pi$ if and only if for every set $A \in \Psi(S)$ and each $a \in \cup_{i \in[k]} \pi_{i}$ the following holds for all $i \in[k]$ such that $\mathrm{Ch}_{T_{i}}(a) \cap A \neq \emptyset$.

(N1) If $a \in S$, then $\left|\mathrm{Ch}_{T_{i}}(a) \cap A\right|=1$.

(N2) If $a \notin S$, then $\mathrm{Ch}_{T_{i}}(a) \subseteq A$.

Proof Consider any $A \in \Psi(S)$. Let $W$ be the connected component of $H_{\mathcal{P}}(\pi) \backslash S$ containing $A$ and let $\pi^{W}$ be the position defined by Eq. (2). To prove the lemma, we show that $\pi^{W}$ is valid if and only if conditions (N7) and (N7) hold.

$(\Longrightarrow)$ Suppose $\pi^{W}$ is valid.

Consider any label $a \in S$ and any $i \in[k]$ such that $\mathrm{Ch}_{T_{i}}(a) \cap A \neq \emptyset$. Since $\pi^{W}$ is valid, $\pi_{i}^{W}=\{b\}$, where $b=\mathrm{LCA}_{T_{i}}\left(X_{i} \cap W\right)$. Thus, $b$ is a minimal label of $T_{i}$ in $X_{i} \cap W$, and so $b \in \mathrm{Ch}_{T_{i}}(a)$. Thus $\left|\mathrm{Ch}_{T_{i}}(a) \cap W\right|=1$, and (N7) holds.

Consider any label $a \in \bigcup_{i \in[k]} \pi_{i} \backslash S$ such that $\mathrm{Ch}_{T_{i}}(a) \cap A \neq \emptyset$. Then, every node in $T_{i}(a)$ must lie inside $W$ and, since $\pi$ is valid, $a$ is minimal in $X_{i} \cap W$. Thus, $\pi_{i}^{W}=\pi_{i}$. Since $a$ remains connected to all its children, $\mathrm{Ch}_{T_{i}}(a) \subseteq A_{j}$, and thus (N7) holds.

( $\Longleftrightarrow$ ) Suppose that for every $a \in \cup_{i \in[k]} \pi_{i}$ and every $i \in[k]$ such that $\mathrm{Ch}_{T_{i}}(a) \cap A \neq \emptyset$, condition (N7) or (N7) holds, depending on whether or not $a \in S$.

Suppose $a \in S$. Consider any $i \in[k]$ such that $\mathrm{Ch}_{T_{i}}(a) \cap A \neq \emptyset$. By (N7), $A$ contains only one child $c \in \mathrm{Ch}_{T_{i}}(a)$. We claim that $c=\mathrm{LCA}_{T_{i}}\left(X_{i} \cap W\right)$. Assume, to the contrary that $W$ contains another label $c^{\prime}$ from $T_{i}(a)$, but $c^{\prime} \notin V\left(T_{i}(c)\right)$. By (N7), $c^{\prime} \notin \mathrm{Ch}_{T_{i}}(a)$. Suppose $c^{\prime}$ is some descendant of another child $b$ of $a$. But $b$ must also be in $W$, contradicting (N7). Therefore, $c$ is the minimal label of $T_{i}$ in $X_{i} \cap W$.

Suppose $a \in \bigcup_{i \in[k]} \pi_{i} \backslash S$. By condition (N7), $V\left(T_{i}(a)\right.$ ) must be contained in $W$, and $a$ is the minimal label of $T_{i}$ in $X_{i} \cap W$ because $a$ is the root of $T_{i}(a)$.

Hence, $\pi^{W}$ is valid, for each connected component $W$ of $H_{\mathcal{P}}(\pi) \backslash S$. Therefore, $S$ is nice.
Suppose $S$ is a nice exposed subset in a valid position $\pi$ and let $A$ be any set in $\Psi(S)$. The position associated with $A$ is the position $\pi^{A}$, where, for each $i \in[k], \pi_{i}^{A}$ is defined as follows. If $\pi_{i}=\emptyset$, then $\pi_{i}^{A}=\emptyset$. Otherwise, let $a$ be the single element in $\pi_{i}$. Then,

$$
\pi_{i}^{A}= \begin{cases}\mathrm{Ch}_{T_{i}}(a) \cap A & \text { if } a \in S, \text { and } \\ \pi_{i} & \text { if } a \notin S .\end{cases}
$$

Consider the profile of Fig. 1, whose display graph is in Fig. 3. Note that

$$
\mathrm{Ch}_{\mathcal{P}}\left(\pi^{\text {init }}\right)=\{2, d, b, c, g, k, 5, f, i, j, l\} .
$$

Let $S=\{1\}$. It can be verified that the partition $\Psi(S)=\{A, B\}$ of $\mathrm{Ch}_{\mathcal{P}}\left(\pi^{\text {init }}\right)$ where

$$
A=\{d\} \text { and } B=\{2, b, c, g, k, 5, f, i, j, l\}
$$

satisfies the conditions of Lemma 7. Thus, $S$ is a nice exposed subset. Using Eq. (3), we obtain

$$
\pi^{A}=(\{d\}, \emptyset, \varnothing, \emptyset) \text { and } \pi^{B}=(\{2\},\{4\},\{g\},\{6\}) .
$$

Observe that $(S, \Pi)$, where $\Pi=\left\{\pi^{A}, \pi^{B}\right\}$, is precisely the good decomposition of position $\pi^{\text {init }}$ presented in the previous section. The next lemma shows that this is not a coincidence.

Lemma 8 Suppose $S$ is a nice exposed subset of $\pi$. Let $\Pi=\left\{\pi^{A}: A \in \Psi(S)\right\}$. Then, $(S, \Pi)$ is a good decomposition of $\pi$.

Proof Let $A$ be any set in $\Psi(S), W$ be the connected component of $H_{\mathcal{P}}(\pi) \backslash S$ that contains $A$, and $\pi^{W}$ be the position defined by Eq. (2). Since $S$ is a nice set, $\pi^{W}$ is valid. To prove the lemma it suffices to show that $\pi^{A}=\pi^{W}$. Consider each $i \in[k]$.

- If $\pi_{i}=\emptyset$, then we have $\pi_{i}^{A}=\pi_{i}^{W}=\emptyset$.

- Now, suppose $\pi_{i}=\{a\}$, for some $a \in \bigcup_{i \in[k]} \pi_{i}$.

- If $a \in S$, then, by Lemma $7, \mathrm{Ch}_{T_{i}}(a) \cap A=\{c\}$, for some $c \in \mathrm{Ch}_{T_{i}}(a)$. Then, $\pi_{i}^{A}=\{c\}$. Since $a \notin W, c$ must be the minimal label of $T_{i}$ in $X_{i} \cap W$. Therefore, $\pi_{i}^{A}=\pi_{i}^{W}=\{c\}$.

- If $a \notin S$, then, by Lemma $7, \mathrm{Ch}_{T_{i}}(a) \subseteq A$. Then, $a \in W$ and, hence, $a$ is the minimal label of $T_{i}$ in $X_{i} \cap W$. Therefore, $\pi_{i}^{A}=\pi_{i}^{W}$.

Thus, $\pi^{A}=\pi^{W}$ as claimed.

Motivated by Lemma 8 , we say that a pair $(S, \Psi(S))$ is a good partition of $\mathrm{Ch}_{\mathcal{P}}(\pi)$ if the pair $(S, \Pi)$ where $\Pi=\left\{\pi^{A}: A \in \Psi(S)\right\}$ is a good decomposition of $\pi$. 


\section{Maximal good decompositions}

A valid position $\pi$ may have many possible nice exposed sets. We are interested in finding a maximal nice exposed subset; that is, a set $S$ such that $S^{\prime} \subseteq S$, for every nice exposed subset $S^{\prime}$ of $\pi$.

Lemma 9 Let $\pi$ be a valid position in a profile $\mathcal{P}$. Then, $\pi$ has a unique maximal nice exposed subset.

To prove Lemma 9, we need an auxiliary result.

Lemma 10 Let $S$ and $S^{\prime}$ be two nice exposed subsets of $\pi$. Then $S^{\prime \prime}=S \cup S^{\prime}$ is also a nice exposed subset of $\pi$.

Proof Since $S$ and $S^{\prime}$ are exposed subsets, so is $S^{\prime \prime}$. By Lemma 7 , the result follows from the next fact.

Fact. Consider any set $A \in \Psi\left(S^{\prime \prime}\right)$ and any label $a \in \bigcup_{i \in[k]} \pi_{i}$. Then, for each $i \in[k]$ such that $\mathrm{Ch}_{T_{i}}(a) \cap A \neq \emptyset,\left|\mathrm{Ch}_{T_{i}}(a) \cap A\right|=1$ if $a \in S^{\prime \prime}$ and $\mathrm{Ch}_{T_{i}}(a) \subseteq A$ if $a \notin S^{\prime \prime}$.

There are two cases to consider.

- Suppose $a \in S^{\prime \prime}$. Then, either $a \in S$ or $a \in S^{\prime}$. Assume without loss of generality that $a \in S$. Set $A$ is contained in some set in $B \in \Psi(S)$. By Lemma 7, $\left|\mathrm{Ch}_{T_{i}}(a) \cap B\right|=1$. Thus, $\left|\mathrm{Ch}_{T_{i}}(a) \cap A\right| \leq 1$. But $\mathrm{Ch}_{T_{i}}(a) \cap A \neq \emptyset$, so $\left|\mathrm{Ch}_{T_{i}}(a) \cap A\right|=1$.

- Suppose $a \notin S^{\prime \prime}$. Since $a \notin S$ and $a \notin S^{\prime}$ and $\mathrm{Ch}_{T_{i}}(a) \cap A \neq \emptyset$, the connected component of
$H_{\mathcal{P}}(\pi) \backslash S^{\prime \prime}$ containing $A$ contains $a$ and thus $\mathrm{Ch}_{T_{i}}(a) \subseteq A$.

Proof of Lemma 9 Suppose, on the contrary, that there exist at least two distinct maximal nice exposed subsets $S, S^{\prime}$. By Lemma 10, $S^{\prime \prime}=S \cup S^{\prime}$ is also a nice exposed subset of $\pi$. But $S \subset S^{\prime \prime}$, contradicting the maximality of $S$.

Corollary 1 Let $\pi$ be a valid position in a profile $\mathcal{P}$ and $S$ be the maximal nice exposed subset of $\pi$. If $\pi$ has an agreement tree, then $S \neq \emptyset$.

Proof Suppose, on the contrary, that $\pi$ has an agreement tree, but $S=\emptyset$. Then, by Lemma 9, every nice exposed subset in $\pi$ must be empty. But, by Lemma 6 , this implies that $\pi$ has no agreement tree, a contradiction.

Let $S$ be the maximal nice exposed subset in $\pi$ and $\Pi$ be the set of successor positions of $\pi$ with respect to $S$. We refer to $(S, \Pi)$ as the maximal good decomposition of $\pi$.

\section{Constructing an agreement tree}

Algorithm BuildAST(Algorithm 1) takes as input a profile $\mathcal{P}$ on a set of labels $X$ and either returns an agreement tree for $\mathcal{P}$ or reports that no such tree exists. BuildAST assumes the availability of an algorithm Decompose, to be described later, that, given a valid position $\pi$ in $\mathcal{P}$, returns a maximal good decomposition $(S, \Pi)$ of $\pi$.

Algorithm 1: Testing agreement

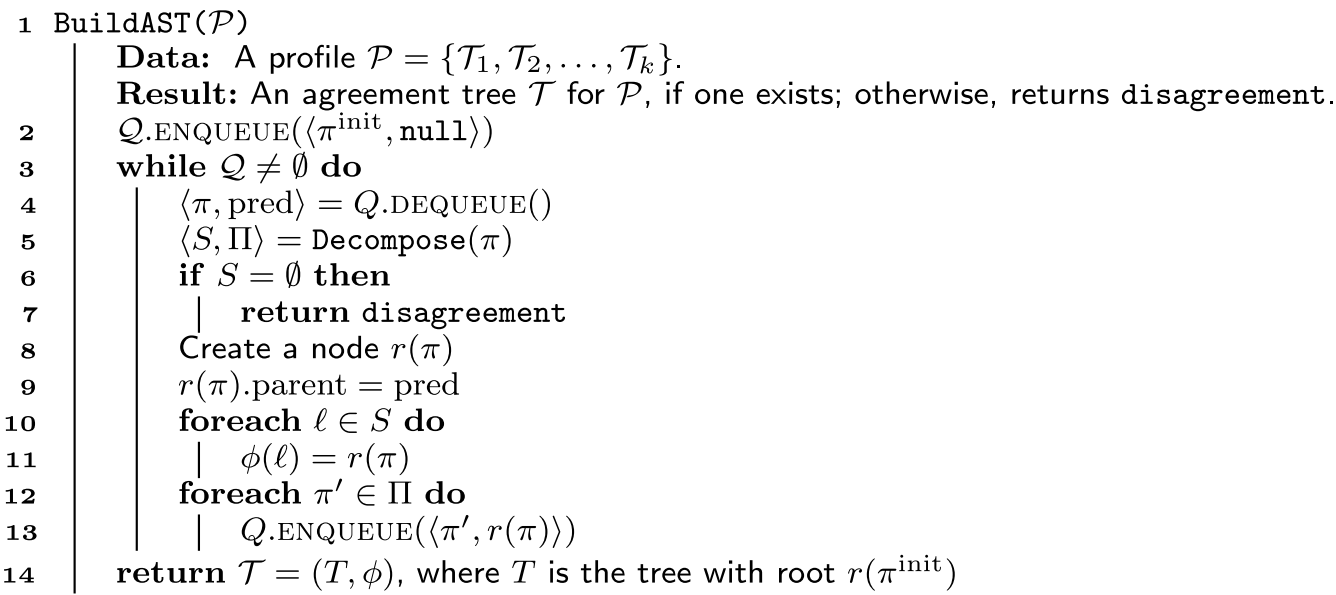


BuildAST proceeds from the top down, starting from the initial position $\pi^{\text {init }}$ of $\mathcal{P}$, attempting to construct an agreement tree for $\mathcal{P}$ in a breadth-first manner. Like other algorithms based on breadth-first search, BuildAST uses a queue, which stores pairs $\langle\pi$, pred $\rangle$ where $\pi$ is a position in $\mathcal{P}$ and pred is a reference to the parent of the tree node (potentially) to be created for $\pi$. At the outset, the queue contains only the pair $\left\langle\pi^{\text {init }}\right.$, null $\rangle$, corresponding to the root of the agreement tree, which has no parent.

At each iteration of its outer while loop (lines 3-13), BuildAST extracts a pair $\langle\pi$, pred $\rangle$ from its queue and invokes Decompose to obtain a maximal good decomposition $(S, \Pi)$ of $\pi$. If $S=\emptyset$, then, by Corollary 1 , no agreement tree for $\pi$ exists. BuildAST reports this fact (line 7) and terminates.

If $S \neq \emptyset$, BuildAST creates a tree node $r(\pi)$ for $\pi$; $r(\pi)$ is the tentative root for the agreement tree for $\pi$. By Lemma 6 , if $\pi$ has an agreement subtree, then it has an agreement tree where $\phi(\ell)=r(\pi)$. Lines 10-11 set up the mapping $\phi$ accordingly. Also by Lemma 6 , if $\pi$ has an agreement tree, then so does each position $\pi^{\prime} \in \Pi$; furthermore, the roots of the trees for each position in $\Pi$ will be the children of $r(\pi)$. Thus, BuildAST adds $\left\langle\pi^{\prime}, r(\pi)\right\rangle$, for each $\pi^{\prime} \in \Pi$ to the queue, to ensure that $\pi^{\prime}$ is processed at a later iteration and that the root of the agreement tree constructed for $\pi^{\prime}$ (if such a tree exists) has $r(\pi)$ as its parent (lines 12-13). Therefore, if BuildAST terminates without reporting disagreement, the result returned in line 14 is an agreement tree for $\mathcal{P}$. BuildAST indeed terminates, because there are only two possibilities at any given iteration: either the algorithm terminates reporting disagreement or (since $S \neq \emptyset)$ the maximal good decomposition $(S, \Pi)$ of $\pi$ has the property that $\bigcup_{\pi^{\prime} \in \Pi} X_{\mathcal{P}}\left(\pi^{\prime}\right)$ is a proper subset of $X_{\mathcal{P}}(\pi)$. The number of iterations of BuildAST cannot exceed the total number of nodes in an agreement tree for $\mathcal{P}$, which is $O(n)$. Thus, we have the following result.

Theorem 1 Given a profile $\mathcal{P}=\left\{\mathcal{T}_{1}, \mathcal{T}_{2}, \ldots, \mathcal{T}_{k}\right\}$, Buil$\mathrm{dAST}$ returns an agreement tree $\mathcal{T}$ for $\mathcal{P}$, if such a tree exists; otherwise, BuildAST returns disagreement. The total number of iterations of BuildAST's outer loop is $O(n)$.

\section{Finding the maximal good decomposition}

Algorithm 2: Computing the maximal good decomposition.

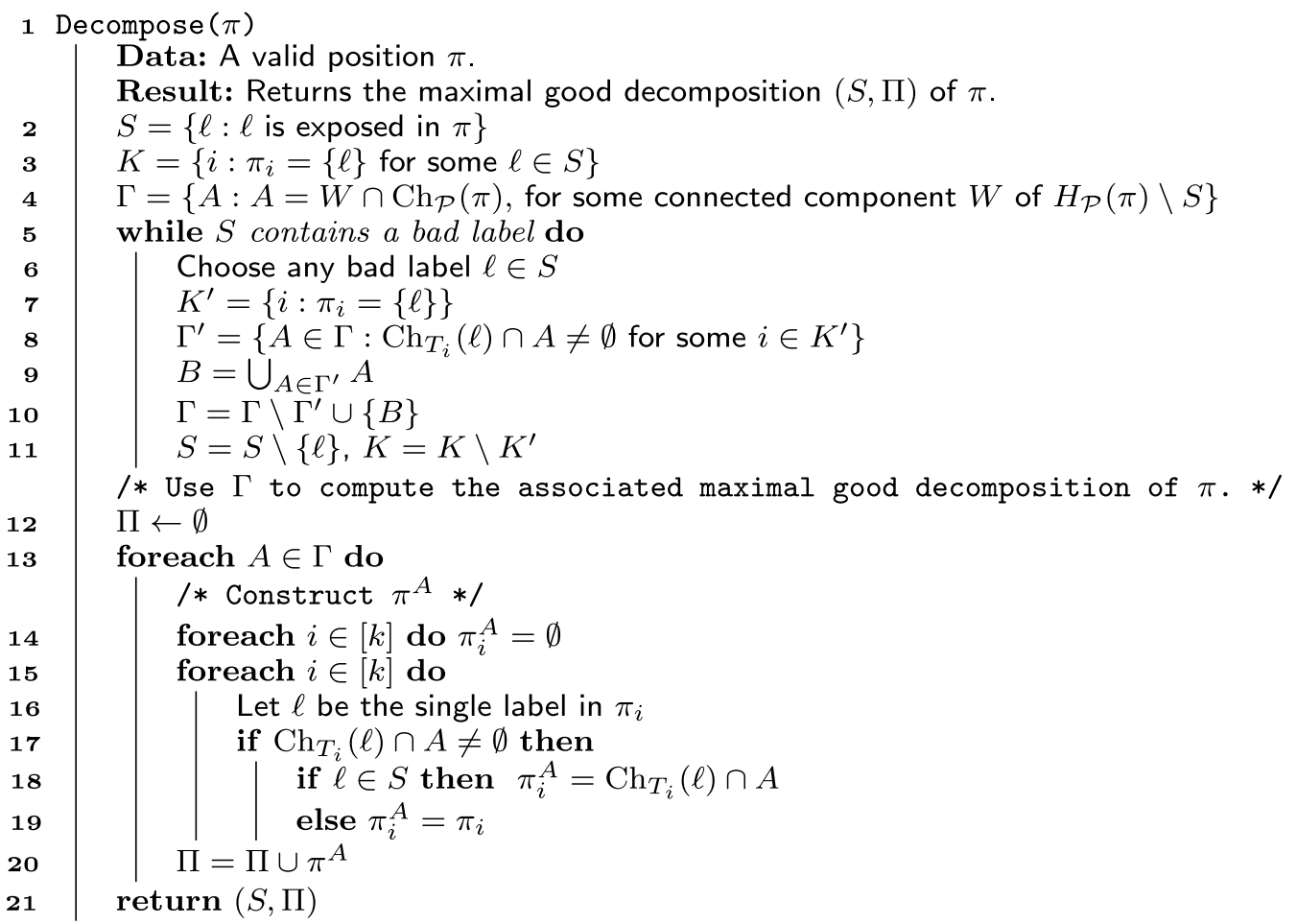


Algorithm Decompose (Algorithm 2) computes a maximal good decomposition of a position $\pi$. Throughout its execution, Decomposemaintains a set $S$ that is a superset of the maximal nice exposed subset of $\pi$ and a partition $\Gamma$ of $\mathrm{Ch}_{\mathcal{P}}(\pi)$. We will argue that before and after every iteration of the while loop of Lines 5-11, $\Gamma=\Psi(S)$. We will also show that, after the loop terminates, $S$ is a maximal nice exposed subset. Lines $12-20$ use $S$ and $\Psi(S)$ to compute the maximal good decomposition of $\pi$. Next, we describe and analyze Decomposein detail.

Lines 2 and 3 of Decomposeinitialize $S$ to contain all exposed labels in $\pi$ and $K$ to consist of the indices of the trees in $\mathcal{P}$ that contain the labels in $S$. Line 11 initializes $\Gamma$ using $H_{\mathcal{P}}(\pi)$. We say that a label $\ell \in S$ is bad if there exist $i \in K$ and $A \in \Gamma$ such that $\pi_{i}=\{\ell\}$ and $\left|\mathrm{Ch}_{T_{i}}(\ell) \cap A\right| \geq 2$. Intuitively, a label $\ell$ is bad if $\ell$ must be a multifurcation in any agreement tree for $\mathcal{P}$, but at least two of $\ell$ 's children lie in the same set in $\Gamma$, while the others lie in different sets.

Lines 5-11 of Decompose construct the maximal nice exposed subset by deleting bad labels from $S$ and merging sets in $\Gamma$ accordingly. Conceptually, removing a bad label from $S$ is equivalent to reinserting it into the graph. Thus, the union operations in the while loop of lines 5-11 can be interpreted as reconnecting bad labels to their children. In the implementation of Decompose, however, labels and the edges to their children are only deleted once. To understand why this is possible, observe that once a label $\ell$ becomes exposed in a position $\pi$, it remains exposed in every position where $\ell$ subsequently appears, until it is finally deleted from the graph or BuildASTterminates. Thus, conceptually, at every call to Decomposewhere $\ell$ is exposed, lines 2-11 add $\ell$ to $S$ and delete $\ell$ from the graph, but then an iteration of lines 5-11 may possibly delete $\ell$ from $S$ and reinsert it into the graph. Instead, our implementation of Decomposedeletes $\ell$ only once. When an iteration of lines 5-11 calls for deleting $\ell$ from $S$, instead of adding $\ell$ back to the graph, we put the various components that would have been reunited into a "virtual" connected component (a similar idea is used in [11]). We elaborate on our approach in the next section.

Lemma 11 Let $\pi$ be a valid position in a profile $\mathcal{P}$ and let $S^{*}$ be the maximal nice exposed subset in $\pi$. Let $S_{j}$ and $\Gamma_{j}$ denote the values of $S$ and $\Gamma$ after $j$ iterations of the loop of Lines 5-11 of Decompose, and $r$ denote the total number of iterations of the loop. Then, $r \leq k, \Gamma_{j}=\Psi\left(S_{j}\right)$, for $j \in\{0,1, \ldots, r\}$, and $S_{0} \supset S_{1} \supset S_{2} \supset \cdots \supset S_{r}=S^{*}$.

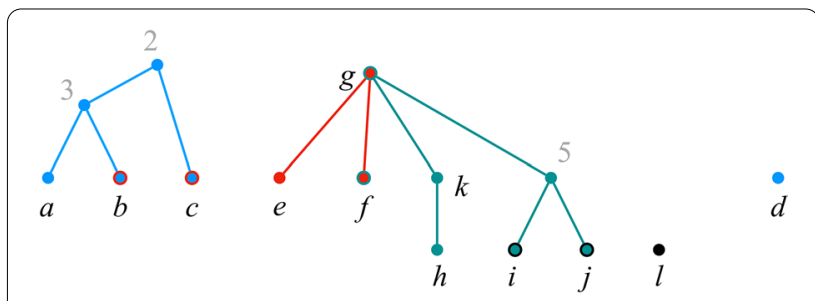

Fig. $5 H_{\mathcal{P}}\left(\pi^{\text {init }}\right) \backslash S$. The set of exposed labels in $\pi^{\text {init }}$ is $S=\{1,4,6\}$

Proof The $j$ th iteration of the loop, $j>1$, removes one bad label from $S_{j-1}$. Thus, $S_{j} \subset S_{j-1}$. Since $|S| \leq k$, the number of iterations is at most $k$.

Let us prove that $\Gamma_{j}=\Psi\left(S_{j}\right)$ and $S_{j} \supseteq S^{*}$, for each $j \in\{0,1, \ldots, r\} . \Gamma_{0}=\Psi\left(S_{0}\right)$ holds by construction and $S_{0} \supseteq S^{*}$ holds trivially. Now assume that $\Gamma_{j-1}=\Psi\left(S_{j-1}\right)$ and $S_{j-1} \supseteq S^{*}$. Note that $S_{j}=S_{j-1} \backslash\{\ell\}$, where $\ell$ is the bad label chosen in line 6 . Since the body of the loop merges all the sets in $\Gamma_{j-1}$ that contain a child of $\ell$, we have $\Gamma_{j}=\Psi\left(S_{j}\right)$. Furthermore, $\ell$ cannot be in $S^{*}$, so $S_{j} \supseteq S^{*}$.

We claim that, for each $j \in\{0,1, \ldots, r\}$, each $\ell \in \bigcup_{i \in[k]} \pi_{i} \backslash S_{j}$, there is an $A \in \Gamma_{j}$ such that $\mathrm{Ch}_{\mathcal{P}}(\ell) \subseteq X_{\mathcal{P}}(A)$. This is true by construction for $j=0$, and the body of the while loop ensures that this remains true throughout the execution of the algorithm.

At termination of the while loop, $S_{r}$ contains no bad labels. Thus, $\Gamma_{r}=\Psi\left(S_{r}\right)$ satisfies the conditions of Lemma 7 with respect to $S_{r}$. Thus, $\left(S, \Psi\left(S_{r}\right)\right)$ is a good partition of $\mathrm{Ch}_{\mathcal{P}}(\pi)$.

When the loop of lines 5-11 terminates, $S_{r}$ is a maximal nice exposed subset in $\pi$. By Lemma $9, S_{r}$ must be the maximal exposed subset, $S^{*}$.

Lines 12-20 of Decomposeuse Eq. (3) to construct the good decomposition $(S, \Pi)$ of $\pi$, where $\Pi=\left\{\pi^{A}: A \in \Psi(S)\right\}$. Thus, by Lemma 8 , we have the following.

Lemma 12 Decompose returns the maximal good decomposition of $\pi$.

Figure 5 shows the graph $H_{\mathcal{P}}\left(\pi^{\text {init }}\right) \backslash S$, from which we conclude that, in line 11 of Decompose, $\Gamma=\Psi(S)=\left\{A_{1}, A_{2}, A_{3}, A_{4}\right\}, \quad$ where $\quad A_{1}=\{2, b, c\}$, $A_{2}=\{d\}, A_{3}=\{f, k, 5, g, i, j\}, A_{4}=\{l\}$. 


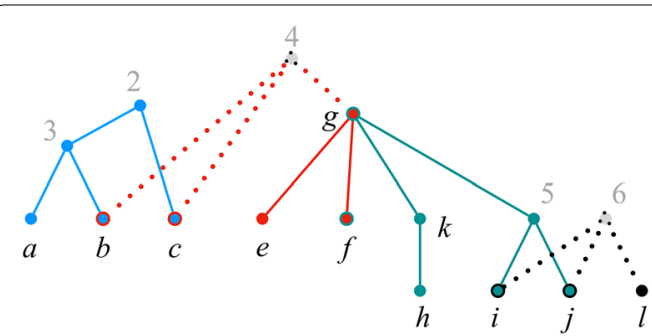

Fig. 6 "Virtual" reconnections. The graph $H_{\mathcal{P}}\left(\pi^{\text {init }}\right) \backslash S$ of Fig. 5 after virtually reconnecting labels 4 and 6

The while loop of lines 5-11 examines each label in set $S$ to identify a bad label. Label 4 is bad, since $\pi_{2}=\{4\}$ and $\left|\mathrm{Ch}_{T_{2}}(4) \cap A_{1}\right|=|\{b, c\}| \geq 2$. Label 6 is also bad, since $\pi_{4}=\{6\}$ and $\left|\mathrm{Ch}_{T_{4}}(6) \cap A_{3}\right|=|\{i, j\}| \geq 2$.

Let us assume that label 6 is processed first. The body of the while loop replaces sets $A_{3}, A_{4} \in \Gamma$ with their union to yield $\Gamma=\Psi(S)=\left\{A_{1}, A_{2}, B\right\}$, where $B=A_{3} \cup A_{4}=\{f, k, 5, g, i, j, l\}$. After this iteration, $S=\{1,4\}$.

In the next iteration, label $\ell=4$ triggers the union of sets $A_{1}$ and $B$, resulting in $\Gamma=\Psi(S)=\left\{A_{2}, B^{\prime}\right\}$, where $B^{\prime}=A_{1} \cup B$, and $S=\{1\}$. After this iteration, $S$ contains no bad labels. Thus, by Lemma 11, $S$ is the maximal nice exposed subset.

The union operations in the while loop can be interpreted as virtually reconnecting the bad labels-labels 4 and 6 in the example-to their children. Figure 6 uses dotted lines to represent such virtual reconnections. Each virtually connected component contains all the labels in precisely one of the sets of the collection $\Gamma$ in the minimal good partition $(S, \Gamma)$ of $\mathrm{Ch}_{\mathcal{P}}\left(\pi^{\text {init }}\right)$. As mentioner earlier, however, for efficiency our algorithm does not actually reconnect deleted labels.

The virtually connected components are also related to the positions in the (maximal) good decomposition of $\mathrm{Ch}_{\mathcal{P}}\left(\pi^{\text {init }}\right)$. Consider the iteration of Lines 13-20 of Decompose that processes set $A=\{2, b, c, f, k, 5, g, i, j, l\} \in \Gamma$. As explained earlier, the inner for all loop (lines 15-19) implements Eq. (3) to construct $\pi^{A}$. The virtually reconnected labels correspond to the indices $i \in[k]$ such that $\pi_{i}^{A}=\pi_{i}^{\text {init }}$. In particular, iterations 2 and 4 of the inner for all loop set $\pi_{2}^{A}=\pi_{2}^{\text {init }}=\{4\}$ and $\pi_{4}^{A}=\pi_{4}^{\text {init }}=\{6\}$, respectively.

\section{Analysis}

Before we analyze BuildAST's running time, we need to specify some implementation details.

- We assume that we use the data structure of Holm et al. [15], known as $H D T$, to maintain the connected components of $H_{\mathcal{P}}$, as nodes and edges are removed from it.

- Let $\ell$ be any label in $X_{\mathcal{P}}$ and let $\mathcal{J}(\ell)=\left\{i \in[k]: \ell \in X_{i}\right\}$. For $i \in \mathcal{J}(\ell)$, we say that $\ell$ is unseen in tree $i$ if BuildAST has not yet reached a position $\pi$, such that $\ell \in \pi_{i}$. BuildAST maintains a list $\ell$.unseen containing all $i \in[k]$ such that $\ell$ is unseen in tree $i$. Initially, $\ell$.unseen $=\mathcal{J}(\ell)$. The first time BuildAST reaches a position $\pi$ such that $\ell \in \pi_{i}$ for some $i \in[k]$, index $i$ is removed from $\ell$.unseen. Label $\ell$ is exposed when $\ell$.unseen $=\emptyset$.

- For each $\pi$ in BuildAst's queue, the set $\operatorname{Ch}_{\mathcal{P}}(\pi)$ is stored as a sparse array $\left(\left(i, \mathrm{Ch}_{T_{i}}\left(\pi_{i}\right)\right): i \in[k]\right.$ and $\left.\left.\mathrm{Ch}_{T_{i}}\left(\pi_{i}\right)\right) \neq \emptyset\right)$. This enables Decompose to access the parts of $\mathrm{Ch}_{\mathcal{P}}(\pi)$ associated with each input tree separately. We use this representation of $\mathrm{Ch}_{\mathcal{P}}(\pi)$ to build similar representations of the sets in the partition $\Gamma$ of $\mathrm{Ch}_{\mathcal{P}}(\pi)$ produced from $H_{\mathcal{P}}(\pi) \backslash S$ in line 11 of Decompose.

- For each label $a \in \mathrm{Ch}_{\mathcal{P}}(\pi)$, we maintain a mapping that returns, in $O(1)$ time, the set $A \in \Gamma$ containing a. During the execution of Decompose's while loop, sets in $\Gamma$ may be merged, and representations of these merged sets must be produced and the mapping from $\mathrm{Ch}_{\mathcal{P}}(\pi)$ to $\Gamma$ must be modified.

Lemma 13 The total time needed to maintain the display graph throughout the entire execution of BuildAST is $\mathcal{O}\left(n k \log ^{2}(n k)\right)$.

Proof Initializing HDT for $H_{\mathcal{P}}$ takes $\mathcal{O}(n k \log (n k))$ time. Each subsequent connectivity query and edge and node deletion takes $\mathcal{O}\left(\log ^{2}(n k)\right)$ amortized time [15].

After the HDT data structure is initialized, no more edge or vertex insertions are performed. Edge deletions take place only in Line 11 of Decompose. There, $H_{\mathcal{P}}(\pi) \backslash S$ is computed by successively deleting the edges from each label $\ell \in S$ to $\mathrm{Ch}_{\mathcal{P}}(\ell)$, and then deleting $\ell$ itself. Some of these deletions may have already been performed for some ancestor position of $\pi$, where $\ell$ was also exposed. We refer to such an exposed label as old. Labels that are exposed for the first time in $\pi$ are new. We only need to delete edges from each new label $\ell$ in $\pi$, and then delete $\ell$ itself; the old labels are skipped. Therefore, each vertex and edge of $H_{\mathcal{P}}$ is deleted at most once. The total number of vertex and edge deletions over the entire execution of BuildAST is thus $\mathcal{O}(n k)$. The time to perform all these deletions is $\mathcal{O}\left(n k \log ^{2}(n k)\right)$. 
The while loop of lines 5-11 of Decompose merges the child sets collected in the set $\Gamma^{\prime}$ constructed in Line 8. As discussed in the proof of Lemma 15, this is done without modifying the display graph.

In the following results, $d_{i}$ denotes the maximum number of children of a node in tree $T_{i}$, for each $i \in[k]$.

Lemma 14 Excluding the time needed to maintain the display graph, Lines 2, 3, and 11 of Decompose take $\mathcal{O}(n k \log (n k))$ time over the entire execution of BuildAST.

Proof To build sets $S$ and $K$ in lines 2 and 3 , we do the following for each $i \in[k]$ such that $\pi_{i} \neq \emptyset$. Suppose $\pi_{i}=\{\ell\}$. If $i \in \ell$.unseen, we delete $i$ from $\ell$.unseen. If $\ell$.unseen becomes empty, then $\ell$ is exposed. Suppose $\pi$ has a parent position $\pi^{*}$. Then, exposed label $\ell \in \pi_{i}$ is new if $\pi_{i} \neq \pi_{i}^{*}$. This step takes $\mathcal{O}(k)$ time per call to Decomposeand $\mathcal{O}(n k)$ over the entire execution of BuildAST.

To construct $\Gamma$ in line 11 , we need to obtain $W \cap \mathrm{Ch}_{\mathcal{P}}(\pi)$ for each connected component $W$ of $H_{\mathcal{P}}(\pi) \backslash S$. We can do this in $\mathcal{O}(n k \log n k)$ time, over the entire execution of BuildAST, using the technique of scanning the smaller component, which has been used for compatibility testing $[11,12]$. Next, we outline the technique.

Let $S_{\text {old }}$ and $S_{\text {new }}$ denote the old and new labels in $S$ at the beginning of an execution of Decompose; thus, $S=S_{\text {old }} \cup S_{\text {new }}$. The labels of $S_{\text {old }}$ and their incident edges have already been deleted. Assume that we know $W \cap \mathrm{Ch}_{\mathcal{P}}(\pi)$ for each connected component $W$ of $H_{\mathcal{P}}(\pi) \backslash S_{\text {old }}$. We consider each node in $S_{\text {new }}$ in succession, deleting its incident edges one at a time. Suppose an edge deletion breaks a component $W$ into two components $W_{1}$ and $W_{2}$, and assume we know $W \cap \mathrm{Ch}_{\mathcal{P}}(\pi)$. We determine whether a label in $W \cap \mathrm{Ch}_{\mathcal{P}}(\pi)$ ends up in $W_{1}$ or $W_{2}$ (thereby obtaining $W_{1} \cap \mathrm{Ch}_{\mathcal{P}}(\pi)$ and $\left.W_{2} \cap \mathrm{Ch}_{\mathcal{P}}(\pi)\right)$ as follows.

Assume without loss of generality that the smaller of $W_{1}$ and $W_{2}$ is $W_{1}$. We initialize $A=\emptyset$ and scan the labels of $W_{1}$. When we scan a label $\ell$ in $W_{1}$, if $\ell \in \mathrm{Ch}_{\mathcal{P}}(\pi)$, we add $\ell$ to $A$ and update $\ell$ 's child mapping to this smaller connected component. After all edge deletions are completed, $W_{1} \cap \mathrm{Ch}_{\mathcal{P}}(\pi)=A$. The set $W_{2} \cap \mathrm{Ch}_{\mathcal{P}}(\pi)$ consists of all labels of $W \cap \mathrm{Ch}_{\mathcal{P}}(\pi)$ that were not moved to $A$. Since a label can be in a smaller component at most $\log _{2}(n k)$ times and there are $\mathcal{O}(n k)$ labels, the total time spent in this process over all deletions performed over the entire execution of BuildASTis $\mathcal{O}(n k \log (n k))$.
Lemma 15 Decompose's while loop takes $\mathcal{O}\left(k \sum_{i \in[k]} d_{i}\right)$ time.

Proof By Lemma 11 the while loop iterates $\mathcal{O}(k)$ times. We complete the proof by showing that each iteration takes $\mathcal{O}\left(\sum_{i \in[k]} d_{i}\right)$ time.

Line 11 of Decomposecomputes $H_{\mathcal{P}}(\pi) \backslash S$ by deleting at most $\sum_{i \in[k]} d_{i}$ edges from $H_{\mathcal{P}}(\pi)$. Therefore,

$$
|\Gamma| \leq \sum_{i \in[k]} d_{i}
$$

For each set $A \in \Gamma$, we maintain a count, initialized to 0 . By Inequality (4), the total time to initialize the counts is $\mathcal{O}\left(\sum_{i \in K} d_{i}\right)$ per iteration. To search for a bad label, for each $i \in K$, we scan each $a \in \mathrm{Ch}_{T_{i}}\left(\pi_{i}\right)$, and increase the count of the set $A$ to which $a$ belongs. If the count for any set $A \in \Gamma$ exceeds one, then $\ell \in \pi_{i}$ is a bad label and the search ends.

Next, we consider the time taken by the body of the while loop. Retrieving $K^{\prime}$ in Line 7 takes constant time. By Inequality (4) and the fact that we have constant-time access to mappings, building $\Gamma^{\prime}$ in line 8 takes $\mathcal{O}\left(\sum_{i \in K^{\prime}} d_{i}\right)$ time as follows. We scan each label $\ell \in \mathrm{Ch}_{T_{i}}\left(\pi_{i}\right)$ for each $i \in[k]$ and retrieve the set $A \in \Gamma$ that contains $\ell$ using the mapping from $\mathrm{Ch}_{\mathcal{P}}(\pi)$ to $\Gamma$. The process takes $\mathcal{O}\left(\sum_{i \in[k]} d_{i}\right)$ time per call to Decompose.

To compute the union of the sets in $\Gamma^{\prime}$ in line 9 , we start by initializing $B$ to the empty set. We then successively consider each $A \in \Gamma^{\prime}$. At each step, we append every child label $\ell$ from a non-empty entry in the representation of $A$ to the corresponding entry in $B$, and change the mapping of $\ell$ to $B$. Given our representation of the sets in $\Gamma$, this process takes $\mathcal{O}\left(\sum_{i \in[k]} d_{i}\right)$ time in each iteration of the while loop.

Updating $\Gamma$ in Line 10 requires removing every $A \in \Gamma^{\prime}$ from $\Gamma$ and then adding $B$. The time spent on updates is $\mathcal{O}\left(\left|\Gamma^{\prime}\right|\right)$, which is $\mathcal{O}\left(\sum_{i \in K^{\prime}} d_{i}\right)$. Finally, updating $S$ in Line 11 takes constant time and updating $K$ takes $\mathcal{O}\left(\left|K^{\prime}\right|\right)$ time.

Theorem 2 BuildAST can be implemented to run in $\mathcal{O}\left(n k\left(\sum_{i \in[k]} d_{i}+\log ^{2}(n k)\right)\right)$ time, where $n$ is the number of distinct taxa in $\mathcal{P}, k$ is the number of trees in $\mathcal{P}$, and $d_{i}$ is the maximum number of children of tree $T_{\dot{b}}$ for $i \in[k]$.

Proof First, consider the total time spent on lines 2-11 of Decomposeover the entire execution of BuildAST. By Lemmas 13 and 14 , the total time spent 
on lines $2-11$ is $\mathcal{O}\left(n k \log ^{2}(n k)\right)$. BuildASTspends $\mathcal{O}\left(n k \sum_{i \in[k]} d_{i}\right)$ time on lines 5-11 of Decomposesince, by Theorem 1, Decompose is invoked $\mathcal{O}(n)$ times and, by Lemma 15 , each invocation spends $\mathcal{O}\left(k \sum_{i \in[k]} d_{i}\right)$ on those lines. Thus, lines 2-11 of Decomposetake $\mathcal{O}\left(n k\left(\sum_{i \in[k]} d_{i}+\log ^{2}(n k)\right)\right)$ time over the entire execution of BuildAST.

Next, consider the foreach loop of lines 13-20. For each set $A \in \Gamma$ considered in that loop, Decomposeconstructs the successor position $\pi^{A}$ in $\mathcal{O}(k)$ time. Since BuildAST generates $\mathcal{O}(n)$ positions, the total time spent on the loop over the entire execution of BuildAST is $\mathcal{O}(n k)$. This time is dominated by the time spent on lines $2-11$.

\section{Experiments}

Here we present our experimental results with a $\mathrm{C}++$ implementation of BuildAST. Our source code is available on Github (https://github.com/researchGit/Agree mentTesting).

As in earlier work [12], we consider two variants of BuildAST. BuildAST(1) uses the original version of the HDT data structure, which involves level promotion. BuildAST(0) uses a much simpler variant of HDT where level promotion is disallowed. (For a description of level promotion, see [15].) In [12] we showed that the simplified graph connectivity data structure outperforms the more complex data structure in the context of tree compatibility.

We performed our experiments on a machine with a 6-core i7 processor and 16 GB memory.

\section{Real data}

We tested our program on three real profiles.

- Spider profile: From Figure 1 of [3]; consists of two input trees with a total of 24 labels.

- Strepsirrhini profile: Studied in [4]; consists of four input trees with a total of 100 labels.

- Phocidae profile: Studied in [3]; contains 15 input trees with 43 labels.

Our program correctly constructs an agreement tree for the Spiders profile and correctly reports that the other two profiles disagree. Since the three real profiles are small, the running times are negligible, whether we use BuildAST(0) or BuildAST(1).

On the Phocidae profile, our program terminates immediately after processing the initial position. Indeed, the display graph of this profile has a complex structure, with several areas of disagreement. For the Strepsirrhini profile, we identified a single position of the display

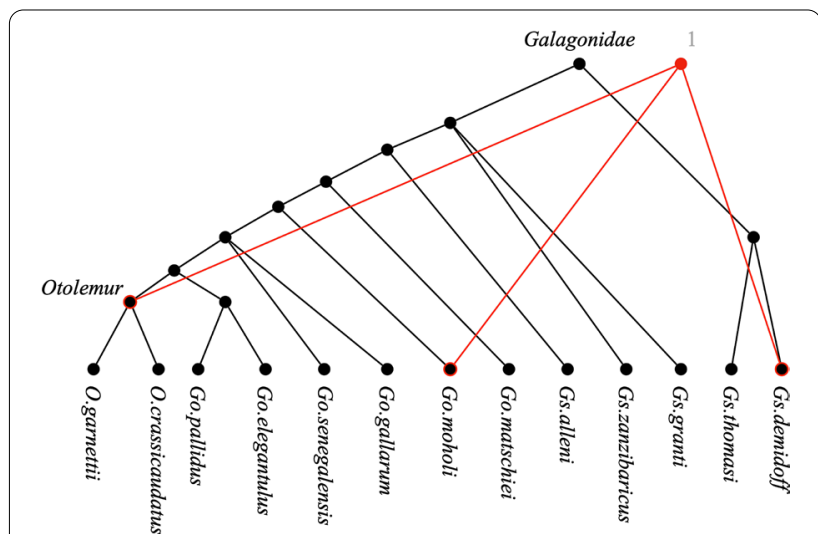

Fig. 7 The part of display graph of the Strepsirrhini profile that leads to disagreement. The black tree and the red tree are subtrees of tree b and tree $\mathbf{a}$, respectively, of Figure 1 in [4]

graph that causes disagreement. Figure 7 shows the region of the display graph corresponding to this position. The region involves taxa from two of the four input trees, colored red and black in the figure. The roots of the corresponding subtree in the black tree is Galagoinidaea, while for the red tree it is an internal node, originally unlabeled, to which we have assigned the artificial label 1. The conflict arises because taxa Otolemur, Go. moholi, and Gs. demidoff are involved in a multifurcation in the red tree, whereas in the black tree the first two taxa are contained in a subtree that does not contain the third. Because of this, after Decomposeinitializes set $S$ to $\{$ Galagoinidaea, 1$\}$, its while loop deletes both labels from $S$, leaving $S=\emptyset$.

\section{Simulated data}

Real profiles, like those considered in the previous section, rarely agree. On such profiles, BuildAST tends to terminate quickly, without processing the input trees in their entirety. To test BuildAST's running time on a wide range of profiles with varying numbers of taxa and trees, we devised an input generator that produces profiles that agree.

\section{Experimental setup}

Given integers $D$ and $m$, the input generator produces a seed tree $\mathcal{T}^{\text {seed }}$ with $m$ labeled nodes, where internal nodes have $D$ children, and where each level except the last is completely filled. Thus, when $D=2$, the seed tree is a complete binary tree. To generate a profile of $k$ trees, we first create a collection of $k$ subsets of labels, $Y_{1}, Y_{2}, \ldots, Y_{k}$. Each subset is obtained by choosing a random number of labels from the set of used labels (the ones chosen so far) and unused labels from the seed tree. From the collection of subsets, we produce a 


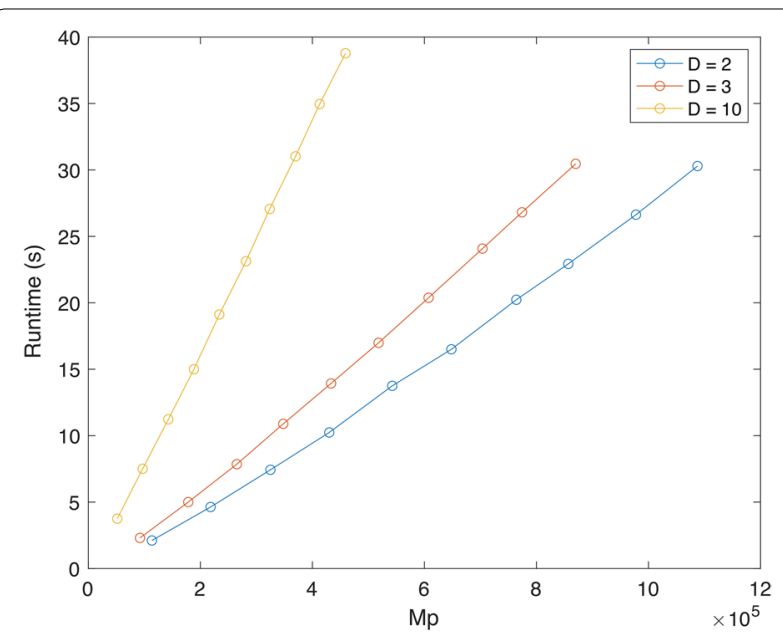

Fig. 8 Running times for trees of degree $D=2,3,10$ in profiles with $k=100$ input trees profile $\mathcal{P}=\left\{\mathcal{T}^{\text {seed }}\left|A_{1}, \mathcal{T}^{\text {seed }}\right| A_{2}, \ldots, \mathcal{T}^{\text {seed }} \mid A_{k}\right\}$. Note that $\mathcal{T}^{\text {seed }} \mid A_{i}$ may contain unlabeled nodes, because certain labels in $A_{i}$ may have a lowest common ancestor in $\mathcal{T}^{\text {seed }}$ that is not in $A_{i}$. We assign such nodes new labels; by Lemma 2, this does not affect agreement.

The reported times are the averages over 30 trials. Times are given in seconds and plotted as a function of $M_{\mathcal{P}}$, the product of the number of taxa and the number of trees; i.e., $M_{\mathcal{P}}=n \cdot k$. Unless stated otherwise, the times reported are for BuildAST(0).

\section{Experiment 1: Fixed number of input trees}

In the first set of experiments, we fix the number of input trees at $k=100$. Since it is difficult to control $n$, the number of taxa, we instead vary the number of labels $m$ in the seed trees from 100 to 1000 with increments of 100 . The number of taxa $n$ falls within a range that depends on $D$. We consider $D=2,3$ and 10 ; the respective ranges of $n$ are $[1135,10875],[922,8701]$ and $[517,5491]$.

Figure 8 shows our results for $D \in\{2,3,10\}$. In all cases, the running time appears to be nearly linear in the number of taxa. This is partly because $\sum_{i \in[k]} d_{i}=k \cdot D$ is fixed. Thus the term $\mathcal{O}\left(n k \sum_{i \in[k]} d_{i}\right)$ in the time bound of Theorem 2 becomes linear in $n$. In theory, then, the $\mathcal{O}\left(n k \log ^{2}(n k)\right)$ dominates the running time. In practice, however, the impact of this term appears to be less significant than the worst-case bound indicates. This seems due to the fact, previously observed in [12], that maintaining dynamic graph connectivity (the source of the polylogarithmic factor) is relatively easy on display graphs.

Figure 9 compares the running times of BuildAST(1) and BuildAST(0) against the theoretical time bound

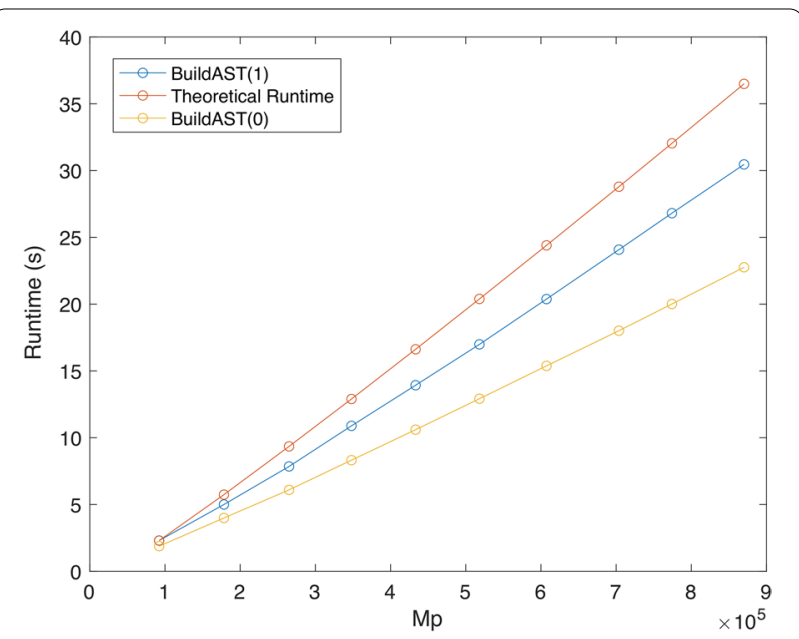

Fig. 9 Theoretical running time versus empirical running time with and without edge promotions for $k=100$ and trees with degree $D=3$

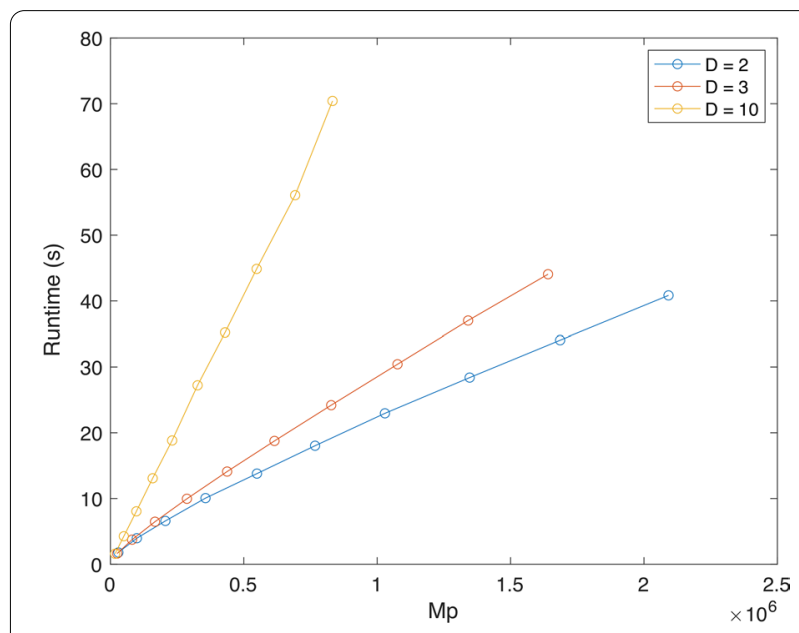

Fig. 10 Running times for profiles of degrees 2, 3, and 10, with $k$ varying from 20 to 200

for input trees with degree $D=3$. The curves show that BuildAST performs well in practice and that BuildAST(0) outperforms BuildAST(1). The latter observation is similar to what we noted in [12].

\section{Experiment 2: Varying the number of input trees}

In the second set of experiments, we varied the number of input trees $k$ from 20 to 200 with increments of 20 , while keeping the number of taxa in the seed trees fixed at $m=500$.

Figure 10 shows that when $D$ equals 2 or 3 , the running time grows sub-linearly at the outset, and then becomes nearly linear. In contrast, when $D=10$, the running time 


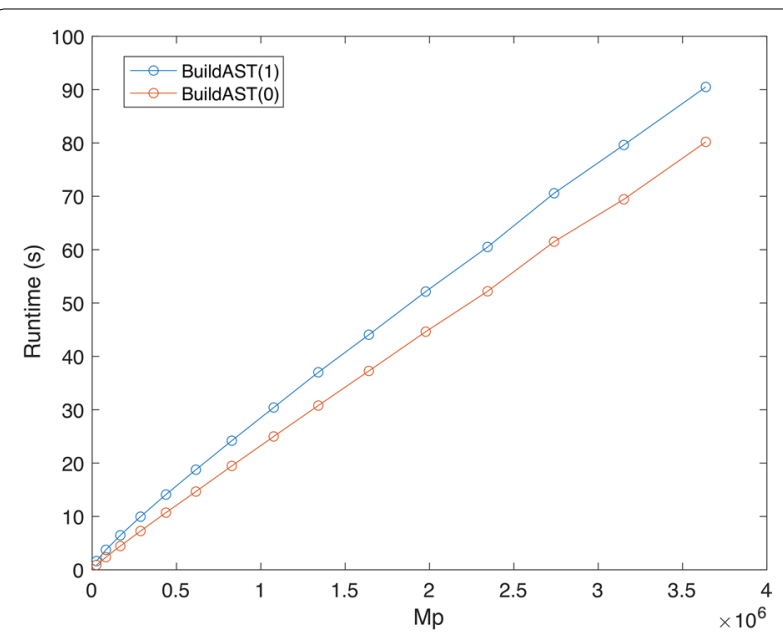

Fig. 11 Running times with and without edge promotion for trees of degree 3 , with $k$ varying from 20 to 300

curve is nearly linear. A possible explanation for these observations centers on the degree to which Decompose's while loop contributes to the overall work. When $D=10$, the input generator produces few bad labels. Thus, the while loop contributes little to the total time. When $D=2$ or 3 , we observe a larger number of bad labels. Since the number of trees $k$ and degree $D$ are small, maintaining graph connectivity initially dominates the total time, but, as the number of trees increases, the while loop again starts to dominate. Thus, one would expect that the running time would be closer to linear for larger numbers of trees. Figure 11, where we extend the number of input trees $k$ to 300, suggests that this is indeed the case. Figure 11, also shows that, as in our first set of experiments, there is no advantage to using level promotion in HDT.

\section{Discussion}

Theorem 2 implies that BuildAST performs well if the sum of the maximum out-degrees is small relative to the number of taxa. In fact, our experiments indicate that BuildAST is faster in practice than Theorem 2 suggests. The reason is that the proof of the theorem assumes the unlikely scenario where every edge deletion performed in constructing $H_{\mathcal{P}}(\pi) \backslash S$ in Decompose generates a new component and that most of these components are remerged in the Decompose's while loop.

The running time of BuildAST can be further improved to $\mathcal{O}\left(n k\left(\sum_{i \in[k]} d_{i}+\log ^{2}(n k) / \log \log (n k)\right)\right)$ using the graph connectivity data structure of reference [27]. It is not clear, however, that the latter data structure

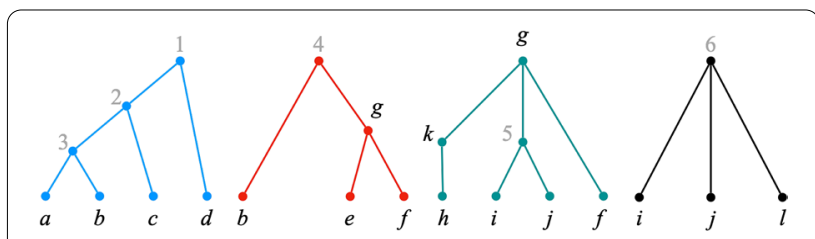

Fig. 12 A profile $\mathcal{P}^{\prime}=\left\{\mathcal{T}_{1}^{\prime}, \mathcal{T}_{2}^{\prime}, \mathcal{T}_{3}^{\prime}, \mathcal{T}_{4}^{\prime}\right\}$. $\mathcal{P}^{\prime}$ is obtained from profile $\mathcal{P}$ of Fig. 1 by removing taxon $\mathrm{c}$ from $\mathcal{T}_{2}$

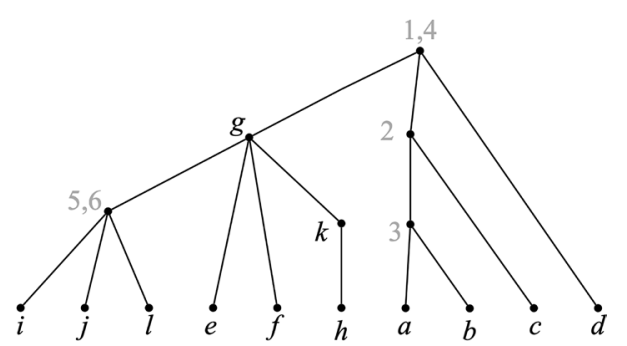

Fig. 13 One possible agreement tree for profile $\mathcal{P}^{\prime}$ of Fig. 12

is practical. In fact, the experiments we present here and in our previous work [12] suggest that data structures much simpler than HDT (and, therefore, than [27]) perform well in practice. These experimental results suggest that a more effective way to speed up BuildAST in practice would be to improve the efficiency with which Decompose deals with bad labels.

If profile $\mathcal{P}$ agrees, BuildASTreturns an agreement tree $\mathcal{T}$ with the property that the set of labels mapped to each node in $\mathcal{T}$ is a maximal nice exposed subset. However, that $\mathcal{P}$ may have other agreement trees that do not have this property. For example, consider the profile $\mathcal{P}$ shown in Fig. 12. Given $\mathcal{P}$ as input, BuildAS Treturns the agreement tree shown in Fig. 13. The tree shown in Fig. 2 (which, as we saw, is an agreement tree for the profile of Fig. 1) is also an agreement tree for $\mathcal{P}$, but the set of labels that map to the root of the tree is not maximal. One open question is whether it is possible to enumerate all agreement trees in time polynomial per agreement tree. A natural way to do this would be to modify Decomposeto enumerate all nice exposed subsets of $\pi$ -not just the maximal one-efficiently. This is equivalent to $\mathrm{Ng}$ and Wormald's approach to enumerating all agreement trees for a profile of leaf-labeled trees [18].

BuildAST can be modified to run in $\mathcal{O}\left(n k \log ^{2}(n k)\right)$ time for profiles $\mathcal{P}$ where the input trees are all binary and solely leaf-labeled. For such profiles, $\left|A \cap \mathrm{Ch}_{T_{i}}\left(\pi_{i}\right)\right| \leq 2$, for $A \in \Gamma$ and $i \in[k]$ in a position $\pi$ of $\mathcal{P}$. Labels $a, a^{\prime} \in \mathrm{Ch}_{T_{i}}\left(\pi_{i}\right)$ are either in the same set $A$ or in different sets $A, A^{\prime}$ where $A, A^{\prime} \in \Gamma$. In the first case, $\ell \in \pi_{i}$ must 
be bad. Bad labels can then be detected earlier in Line 11 and directly removed from $S$. Thus, we can skip Decompose's while loop. Hence, maintaining graph connectivity would dominate the performance of BuildAST.

\section{Conclusions}

BuildAST enables users to deal with hard polytomies. In applications, we may encounter both hard and soft polytomies. It would be interesting to modify Buil$\mathrm{dAST}$ to handle a mixture of both types polytomies, as appropriate.

\section{Abbreviation}

HDT: The dynamic graph connectivity data structure of Holm, de Lichtenberg, and Thorup [15].

\section{Acknowledgements}

The reviewers provided many insightful comments that helped to improve the manuscript. The authors thank Vincent Berry and Charles Semple for providing the Strepsirrhini profile studied in [3]. DFB thanks the College of Liberal Arts and Science, lowa State University, for its support through the Dale D. Grosvenor Chair.

\section{Authors' contributions}

$\mathrm{LL}$ developed the algorithms, with assistance from DFB, his dissertation advisor. LL implemented the algorithms and conducted the experiments, under DFB's supervision. DFB and LL jointly wrote the paper. Both authors read and approved the final manuscript.

\section{Funding}

Not applicable.

\section{Availability of data and materials}

The code used to generate the artificial datasets analyzed in our experiments is in our Github repository. The Strepsirrhini data set was obtained from the authors of [4]. The other two real data sets are publicly available.

\section{Declarations}

Ethics approval and consent to participate

Not applicable.

\section{Consent for publication}

Not applicable.

\section{Competing interests}

The authors declare that they have no competing interests.

Received: 29 April 2021 Accepted: 13 November 2021

Published online: 04 December 2021

\section{References}

1. Aho Alfred V, Sagiv Yehoshua, Szymanski Thomas G, Ullman Jeffrey D. Inferring a tree from lowest common ancestors with an application to the optimization of relational expressions. SIAM J Comput. 1981;10(3):405-21.

2. Baum BR. Combining trees as a way of combining data sets for phylogenetic inference, and the desirability of combining gene trees. Taxon. 1992:41:3-10

3. Berry $V$, Bininda-Emonds $O R$, Semple C. Amalgamating source trees with different taxonomic levels. Syst Biol. 2012;62(2):231-49.

4. Berry V, Semple C. Fast computation of supertrees for compatible phylogenies with nested taxa. Syst Biol. 2006;55(2):270-88.
5. Bininda-Emonds ORP, editor. Phylogenetic Supertrees: Combining Information to Reveal the Tree of Life, vol. 4. Series on Computational Biology. Berlin: Springer; 2004.

6. Bordewich M, Evans G, Semple C. Extending the limits of supertree methods. Anna Comb. 2006;10:31-51.

7. Bryant D, Lagergren J. Compatibility of unrooted phylogenetic trees is FPT. Theor Comput Sci. 2006:351:296-302.

8. Daniel P, Semple C. Supertree algorithms for nested taxa. In: BinindaEmonds ORP, editor. Phylogenetic supertrees: combining information to reveal the Tree of Life. Dordrecht: Kluwer; 2004. p. 151-71.

9. Deng Y, Fernández-Baca D. An efficient algorithm for testing the compatibility of phylogenies with nested taxa. Algorithms Mol Biol. 2017;12:7.

10. Deng Y, Fernández-Baca D. Fast compatibility testing for rooted phylogenetic trees. Algorithmica. 2018:80(8):2453-77.

11. Fernández-Baca D, Guillemot S, Shutters B, Vakati S. Fixed-parameter algorithms for finding agreement supertrees. SIAM J Comput. 2015:44(2):384-410.

12. Fernández-Baca D, Liu L. Tree compatibility, incomplete directed perfect phylogeny, and dynamic graph connectivity: an experimental study. Algorithms. 2019;12(3):53.

13. Fernández-Baca $D$, Liu L. Testing the agreement of trees with internal labels. In: Zhipeng C, Ion M, Giri N, Pavel S, Xuan G, editors. Bioinformatics research and applications. Cham: Springer; 2020. p. 127-39.

14. Hinchliff CE, Smith SA, Allman JF, Burleigh JG, Chaudhary R, Coghill LM, Crandall KA, Deng J, Drew BT, Gazis R, Gude K, Hibbett DS, Katz LA, Laughinghouse IV HD, McTavish EJ, Midford PE, Owen CL, Reed RH, Reesk JA, Soltis DE, Williams T, Cranston KA. Synthesis of phylogeny and taxonomy into a comprehensive tree of life. Proc Natl Acad Sci. 2015:112(41):12764-9.

15. Holm J, de Lichtenberg K, Thorup M. Poly-logarithmic deterministic fullydynamic algorithms for connectivity, minimum spanning tree, 2-edge, and biconnectivity. J ACM. 2001;48(4):723-60.

16. Jansson J, Lingas A, Rajaby R, Sung W-K. Determining the consistency of resolved triplets and fan triplets. In International Conference on Research in Computational Molecular Biology, pages 82-98. Springer, 2017.

17. Maddison WP. Reconstructing character evolution on polytomous cladograms. Cladistics. 1989;5:365-77.

18. Ng MP, Wormald NC. Reconstruction of rooted trees from subtrees. Discrete Appl Math. 1996;69(1-2):19-31.

19. Page RM. Taxonomy, supertrees, and the tree of life. In: Bininda-Emonds OR, editor. Phylogenetic supertrees: combining information to reveal the tree of life. Dordrecht: Kluwer; 2004. p. 247-65.

20. Ragan MA. Phylogenetic inference based on matrix representation of trees. Mol Phylogenet Evol. 1992;1:53-8.

21. Redelings BD, Holder MT. A supertree pipeline for summarizing phylogenetic and taxonomic information for millions of species. PeerJ. 2017;5:e3058.

22. Sanderson MJ. Phylogenetic signal in the eukaryotic tree of life. Science. 2008:321(5885):121-3.

23. Sayers EW, et al. Database resources of the National Center for Biotechnology Information. Nucleic Acids Res. 2009;37(Database issue):D5-15.

24. Semple C, Steel M. Phylogenetics. Oxford: Oxford Lecture Series in Mathematics. Oxford University Press; 2003.

25. The Angiosperm Phylogeny Group. An update of the Angiosperm Phylogeny Group classification for the orders and families of flowering plants: APG IV. Bot J Linnean Soc. 2016;181:1-20.

26. Warnow T. Supertree construction: opportunities and challenges. Technical Report arXiv:1805.03530, ArXiV, May 2018.

27. Wulff-Nilsen C. Faster deterministic fully-dynamic graph connectivity. In: Proceedings of the Twenty-fourth Annual ACM-SIAM Symposium on Discrete Algorithms, SODA '13, pages 1757-1769, Philadelphia, PA, USA, 2013. Society for Industrial and Applied Mathematics.

\section{Publisher's Note}

Springer Nature remains neutral with regard to jurisdictional claims in published maps and institutional affiliations. 